\title{
Fluorsilyl- und Fluorboryl-substituierte Cyclotetrasilazane Synthese und Kristallstrukturen
}

\author{
K. Dippel, U. Klingebiel*, T. Kottke, F. Pauer, G. M. Sheldrick \\ und D. STALKE
}

Göttingen, Institut für Anorganische Chemie der Universität

Inhaltsübersicht. Mono- und dilithiiertes Oetamethylcyclotetrasilazan $(\mathbf{1}, \mathbf{6})$ reagieren mit Fluorsilanen bzw. $\mathrm{F}_{2} \mathrm{BN}\left(\mathrm{SiMe}_{3}\right)_{2}$ unter Erhalt des Cyclotetrasilazangerüstes zu den mono- und disubstituierten Verbindungen $\mathbf{2 - 5}$ und 7-13. Bei der Umsetzung des Dilithiumsalzes (6) mit einem Fluorsilan im Molverhältnis von 1:1 werden die Bicyclen 14 und 15 erhalten. Kristallographische Untersuchungen von $\mathbf{2}, \mathbf{3}, 8$ und 9 zeigen, daß der monosilylierte Achtring in einer Wannen-, der disilylierte Achtring in einer Sesselkonformation vorliegt. In der Kristallstruktur des Bicyclus 14 zeigen die Stickstoffatome der $\mathrm{Si}_{3} \mathrm{~N}$-Einheiten eine pyramidale Umgebung.

Fluorosilyl and Fluoroboryl Substituted Cyclotetrasilazanes. Synthesis and Crystal Structures

Abstract. Mono- and dilithiated octamethylcyclotetrasilazanes $(\mathbf{1}, \mathbf{6})$ react with fluorosilanes and $\mathrm{F}_{2} \mathrm{BN}\left(\mathrm{SiMe}_{3}\right)_{2}$, resepetively, under retention of the cyelotetrasilazane structure yielding the mono- and disubstituted compounds $2-5$ and $7-13$. In the reaction of the dilithium compound 6 with a fluorsilane in equivalent amounts, the bicyclic compounds 14 and 15 are obtained. Crystallographic investigations of the compounds $2,3,8$, and 9 indicate that the monosilylated eight-membered ring has a cradle conformation while the disilylated ring has a chair conformation. The X-ray structure of the bicyclic system 14 indicates that the nitrogen atoms of the $\mathrm{Si}_{3} \mathrm{~N}$-units have a pyramidal environment.

\section{Einfiihrung}

Bei der Ammonolyse von Dimethyldichlorsilan wird zu ungefähr gleichen Anteilen das Hexamethylcyclotrisilazan und das Octamethylcyclotetrasilazan gebildet [1].

Die Chemie dieser beiden leicht zugänglichen Cyclosilazane war lange Zeit stark eingeschränkt. Aufgrund der leichten Spaltbarkeit der Si-N-Bindung reagieren sie mit den meisten Reaktionspartnern unter Bildung acyclischer Verbindungen, Ringkontraktionen und -erweiterungen [2]. Erst die Verwendung von Fluorsilanen als Reaktionspartner ermöglichte es, am Hexamethylcyclotrisilazan unter Erhalt des Sechsringes systematisch Mono-, Di- und Trisubstitutionen durchzuführen [3]. Vom Octamethylcyclotetrasilazan waren vierzig Jahre nach der erstmaligen Synthese keine Reaktionen unter Erhalt des Cyclotetrasilazangerüstes bekannt. 
Das Ziel der hier vorgestellten Arbeit war, durch die Wahl geeigneter Reaktionsbedingungen und Substituenten erste Reaktionen am Octamethylcyclotetrasilazan unter Erhalt des $\mathrm{Si}-\mathrm{N}$-Achtringgerüstes durchzuführen. Kristallographische Untersuchungen von Vertretern dieser neuen Verbindungsklasse sollten dabei Aufschluß über den Einfluß des Silylsubstituenten auf die Konformation des Cyclotetrasilazans geben.

\section{Ergebnisse und Diskussion}

Die Substitutionsreaktionen werden über die Lithiumverbindungen des Octamethylcyclotetrasilazans (OMCTS) durchgeführt. Das Lithiumsalz 1 reagiert bei einer Reaktionstemperatur von $-50^{\circ} \mathrm{C}$ mit Trifluorsilanen und Difluordimethylsilan unter Erhalt des $\mathrm{Si}-\mathrm{N}$-Achtringgerüstes zu den monosubstituierten Verbindungen $\mathbf{2}-\mathbf{5}$.

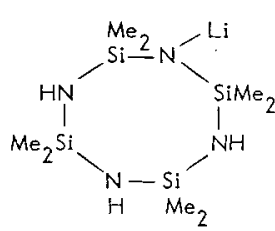

了

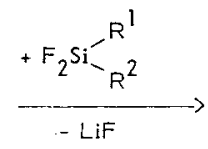

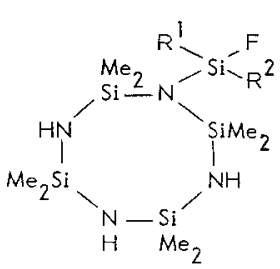

$2-5$

$$
\mid \begin{aligned}
& +1 \\
& +F_{2} S_{i}-R^{i} \\
& -\left(M e_{2} S i-N H\right)_{4} \\
& -L i F
\end{aligned}
$$

\begin{tabular}{l|ll} 
& $\mathrm{R}^{\mathrm{l}}$ & $\mathrm{R}^{2}$ \\
\hline 2 & $\mathrm{~F}$ & $\mathrm{Me}$ \\
3 & $\mathrm{Me}$ & $\mathrm{Me}$ \\
4 & $\mathrm{~F}$ & $\mathrm{Ph}$ \\
5 & $\mathrm{~F}$ & $\mathrm{CMe}_{3}$
\end{tabular}

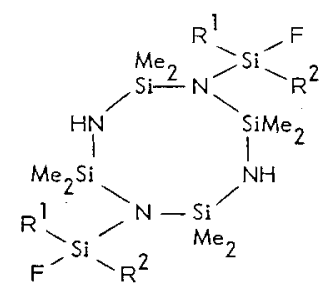

Die Ausbeuten an Monosubstitutionsprodukt sind mit $5-10 \%$ sehr niedrig. Als weitere Produkte werden die entsprechenden disubstituierten Verbindungen und das OMCTS erhalten.

Die Bildung des Disubstitutionsproduktes ist auf eine Umlithiierungsreaktion nach Erhalt der monosubstituierten Verbindung zurückzuführen. Der Fluorsilylsubstituent bewirkt eine Aktivierung des Ringsystems, so daß noch in Lösung vorhandenes Lithiumsalz unter Rückbildung von OMCTS den monosubstituierten Ring erneut lithiiert. Analoge Umlithiierungsreaktionen werden bei der Reaktion von lithiierten Hexamethylcyclotrisilazan mit Fluorsilanen bei erhöhter Reaktionstemperatur beobachtet [4]. 
Die ablaufenden Reaktionen werden durch die Faktoren Temperatur und. Lösungsmittel beeinflußt. Hohe Temperaturen fördern den Umlithiierungsmechanismus, es wird in zunehmendem Anteil die disubstituierte Verbindung gebildet. Gleichzeitig werden durch Erhöhung der Temperatur Isomerisierungsreaktionen begünstigt [5]. Nucleophile Lösungsmittel (THF, Diethylether) fördern ebenfalls die auftretenden Nobenreaktionen.

Die Umsetzung der Dilithiumverbindung 6 mit Fluorsilanen im molaren Verhältnis von $1: 2$ bei $-40^{\circ} \mathrm{C}$ ermöglicht die direkte Synthese von Bis(fluorsilyl)substituierten Cyclotetrasilazanen [6].

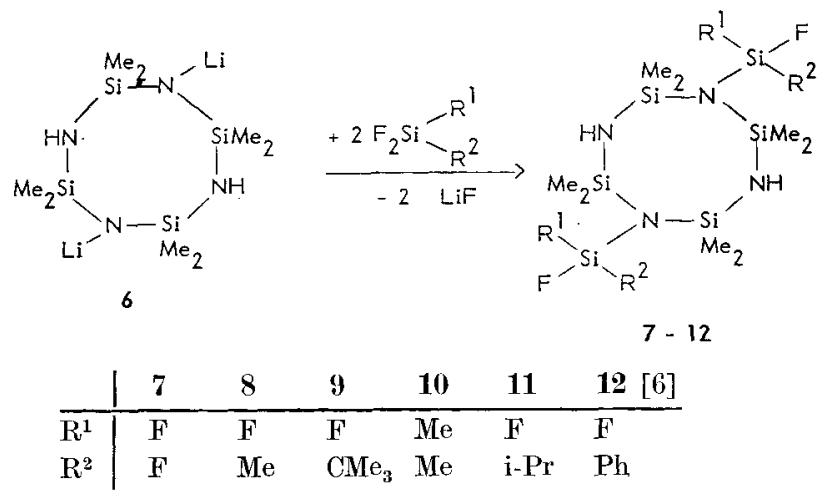

Die Ausbeuten an 7-12 betragen bei dieser Reaktion zwischen $80-90 \%$. Eine Erhöhung der Reaktionstemperatur begünstigt auch hier die Ringkontraktion [5]. Analog der Darstellung Fluorboryl-substituierter Cyclotrisilazane [7] reagiert 6 mit Bis(trimethylsilyl)amino-difluorboranen zu dem disubstituierten Cyclotetrasilazan 13.

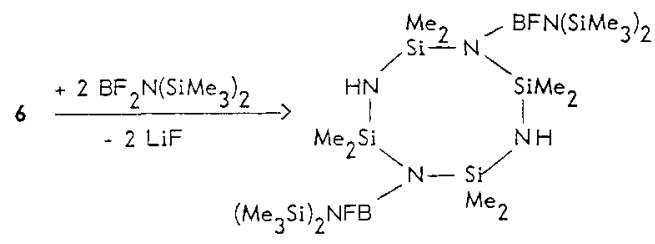

13
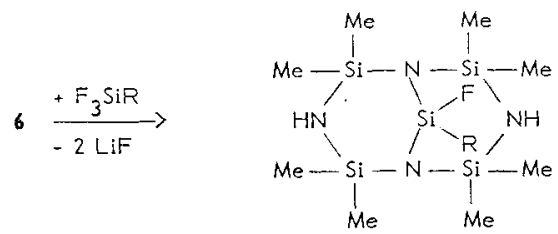

14,15

$$
\begin{aligned}
& 14 \mathrm{R}=\mathrm{Ph}[16] \\
& 15 \mathrm{R}=\mathrm{CMe}_{3}
\end{aligned}
$$

Bei der Umsetzung der Dilithiumverbindung 6 mit Fluorsilanen im Molverhältnis von 1:1 wird das $\mathrm{Si}-\mathrm{N}$-Achtringsystem über ein Siliciumatom überbrückt 
[6]. Die Reaktion mit Phenyltrifluorsilan bzw. tert.-Butyltrifluorsilan führt zu den bicyclischen Systemen 14 und $\mathbf{1 5}$.

Die Verbindungen $2-5$ und $7-15$ sind kristalline Festkörper, die im Hochvakuum unzersetzt durch Destillation oder Sublimation gereinigt werden können.

Kristallstrukturen der monosubstituierten Cyclotetrasilazane 2 und 3

Der Difluormethylsilyl-substituierte Achtring 2 liegt in einer Wannenkonformation vor (Abb. 1). Die Atome Si1, N1, Si3, N3 bzw. Si2, N2, Si4, N4 liegen mit einer mittleren Abweichung von 0,17 pm bzw. 2,94 pm jeweils in einer Ebene; die zwei durch diese Atome definierten Ebenen sind annähernd koplanar. Bedingt durch den - I-Effekt des Fluorsilylsubstituenten ist der exocyclische Si Abstand mit 168,5 pm stark verkürzt. Demzufolge sind die endocyclischen $\mathrm{Si}-\mathrm{N}$ Abstände am N1-Atom mit 177,8 pm (Si1) bzw. 176,1 pm (Si2) deutlich länger als die übrigen $\mathrm{Si}-\mathrm{N}$-Ringbindungen. Die $\mathrm{Si}-\mathrm{N}-\mathrm{Si}$-Winkel am N2-, N3- und N4-Atom liegen im Bereich von $131,6^{\circ}\left(2,7^{\circ}\right)$ und entsprechen damit den Bindungswinkeln im OMCTS [8]. Die drei Si-N-Si-Winkel am N1-Atom weichen nur geringfügig von $120^{\circ} \mathrm{ab}$. Die Ringwinkelverkleinerung am N1-Atom kann mit dem größeren Raumbedarf einer Silylgruppe im Vergleich zu einem Wasserstoffatom erklärt werden.

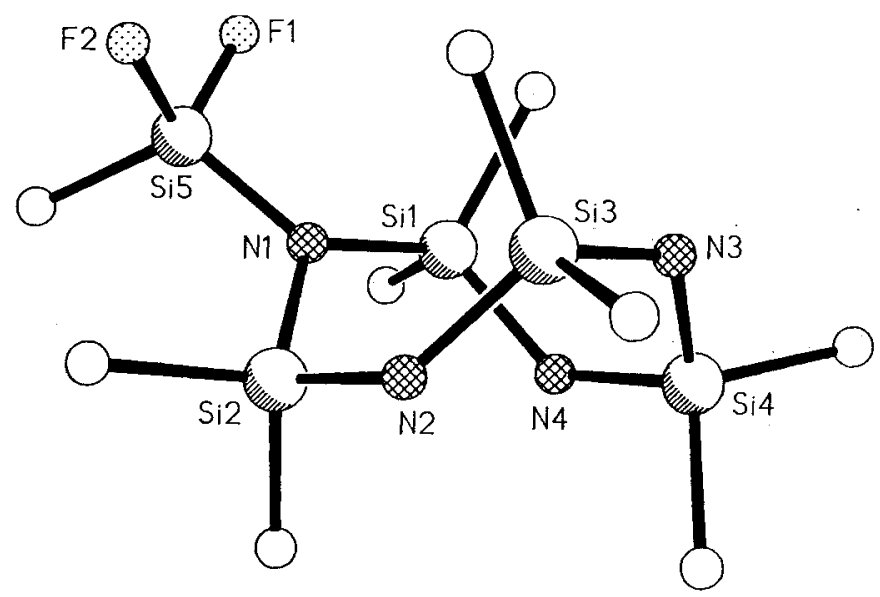

Abb. 1 Kristallstruktur von $\mathbf{2}$

Die Koplanarität der oben genannten Atomquartette kommt dadurch zustande, daß das Molekül im Vergleich zur Wannenkonformation des OMCTS [8] noch weiter um die N1 $\leftrightarrow$ N3-Achse verdreht ist. Dies ist auf das Bestreben des Silylsubstituenten, sich zur Minimierung sterischer Wechselwirkungen vom Zentrum des Ringes zu entfernen, zurückzuführen.

Im Kristall von 2 wird die Ausbildung von intermolekularen Wasserstoffbrücken beobachtet. Jedes Molekül ist über die beiden Fluoratome und zwei 
N-H-Wasserstoffatome mit vier anderen Molekülen verbrückt (Abb. 2). An der Brückenbildung sind jeweils diejenigen Wasserstoffatome beteiligt, die an im Ring gegenüberliegende Stickstoffatome gebunden sind. Hieraus resultiert ein Anordnung in Schichten mit rautenförmigem Muster. Die Länge der ausgebildeten Wasserstoffbrücken ist mit 224 pm (F1) bzw. 231 pm (F2) sehr kurz.

Die Kristallstruktur des Dimethylfluorsilyl-substituierten Achtringes $\mathbf{3}$ ist der Struktur von 2 sehr ähnlich (Abb. 3). Auch 3 nimmt im Kristall eine Wannenkonformation ein. Die Bindungswinkel zeigen im Vergleich zu 2 keine signifikanten Unterschiede. Am N1-Atom wird auch hier eine Ringwinkelverkleinerung beob-

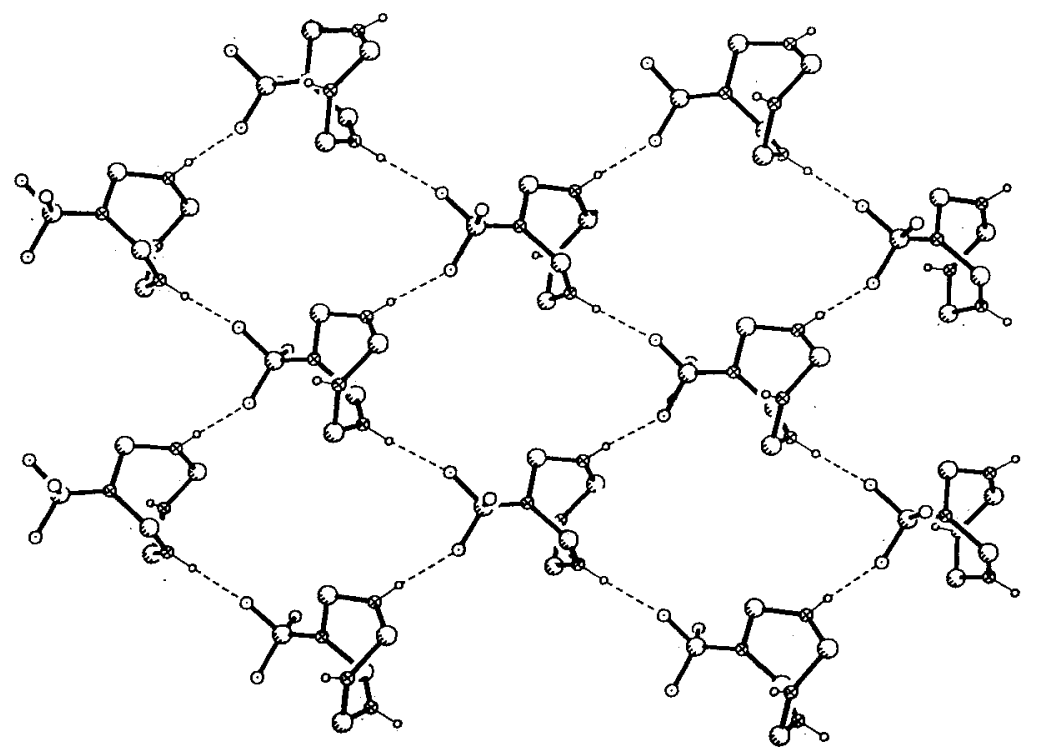

Abb. 2 Anordnung von 2 im Kristall

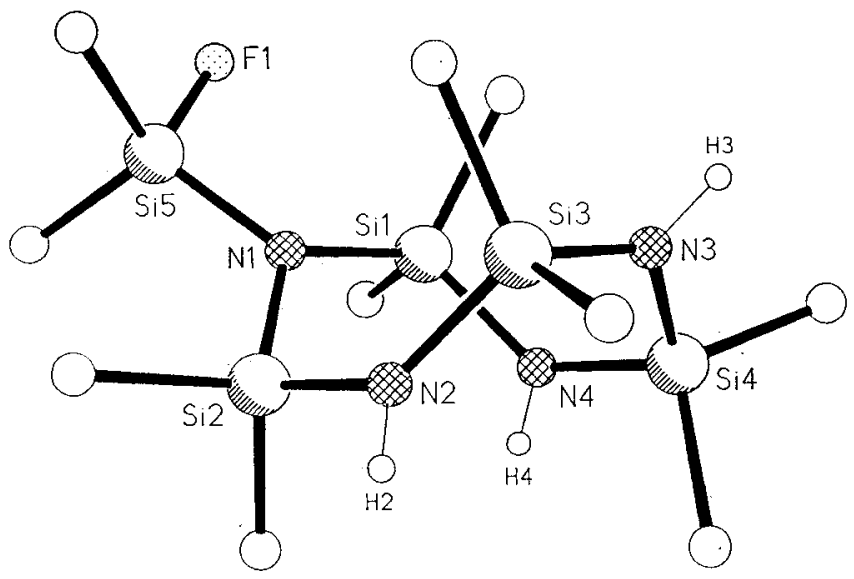

Abb. 3 Kristallstruktur von 3 
achtet. Die Si-N-Bindungsabstände von 3 zeigen jedoch im Bereich des N1-Atoms wesentliche Unterschiede zu 2. Eine Gegenüberstellung dieser Daten verdeutlicht den Einfluß unterschiedlicher Fluorsilylsubstituenten auf die $\mathrm{Si}-\mathrm{N}$-Abstände am silyl-substituierten Stickstoffatom.

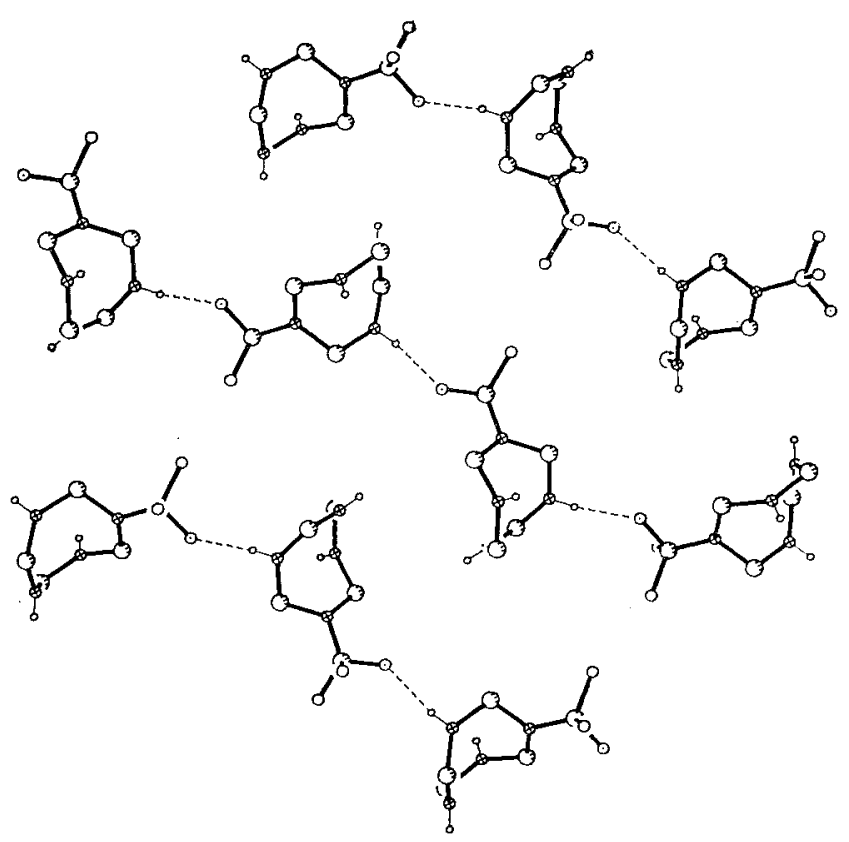

Abb. 4 Anordnung von $\mathbf{3}$ im Kristall

Vergleich der Bindungsabstände [pm] am N1-A
\begin{tabular}{l|lll} 
& N1-Si5 & N1-Si1 & N1-Si2 \\
\hline $\mathbf{2}$ & $168,2(2)$ & $177,8(2)$ & $176,1(2)$ \\
$\mathbf{3}$ & $172,1(2)$ & $176,6(2)$ & $174,7(2)$
\end{tabular}

Da in 3 an das Si5-Atom nur ein elektronenziehendes Fluoratom gebunden ist, wird die Si5-N1-Bindung weniger stark polarisiert, als dies bei 2 der Fall ist. Dies führt zu einer vergleichsweise längeren exocyclischen $\mathrm{Si}-\mathrm{N}$-Bindung in 3 . Dementsprechend sind die endocyclischen $\mathrm{Si}-\mathrm{N}$-Abstände am N1-Atom in 3 kürzer als die entsprechenden Abstände in 2.

Auch bei 3 werden intermolekulare Wasserstoffbrücken im Kristall ausgebildet (Abb. 4). Da pro Molekül nur ein Fluoratom zur Verfügung steht, sind die Moleküle kettenartig angeordnet. Die Verbrückung der Moleküle erfolgt, wie auch in Struktur 2, über eine dem silylsubstituierten Stickstoffatom benachbarte N-HGruppe. Die Länge der H-F-Brücken beträgt für 3236 pm. 


\section{Kristallstrukturen der disubstituierten Cyclotetrasilazane 8 und 9}

Das Bis(difluormethylsilyl)-substituierte OMCTS 8 und das Bis(tert.-butyldifluorsilyl)-substituierte OMCTS 9 zeigen keine signifikanten Unterschiede in der Molekülstruktur. Die Strukturen von 8 und 9 werden deshalb gemeinsam beschrieben und diskutiert.

Der Si-N-Achtring liegt in einer Sesselkonformation vor. Diese wird durch die Transstellung der exocyclischen Silylgruppen begünstigt. Im Zentrum des Achtringes befindet sich ein kristallographisches Inversionszentrum, so daß sich nur eine Molekülhälfte in der asymmetrischen Einheit befindet. Die $\mathrm{Si}-\mathrm{N}-\mathrm{Ab}$ stände der Fluorsilyl-substituierten Stickstoffatome sind auch hier stark unterschiedlich. Die exocyclische N1-Si3-Bindung ist mit 168,5 pm (8) bzw. 169,5 pm

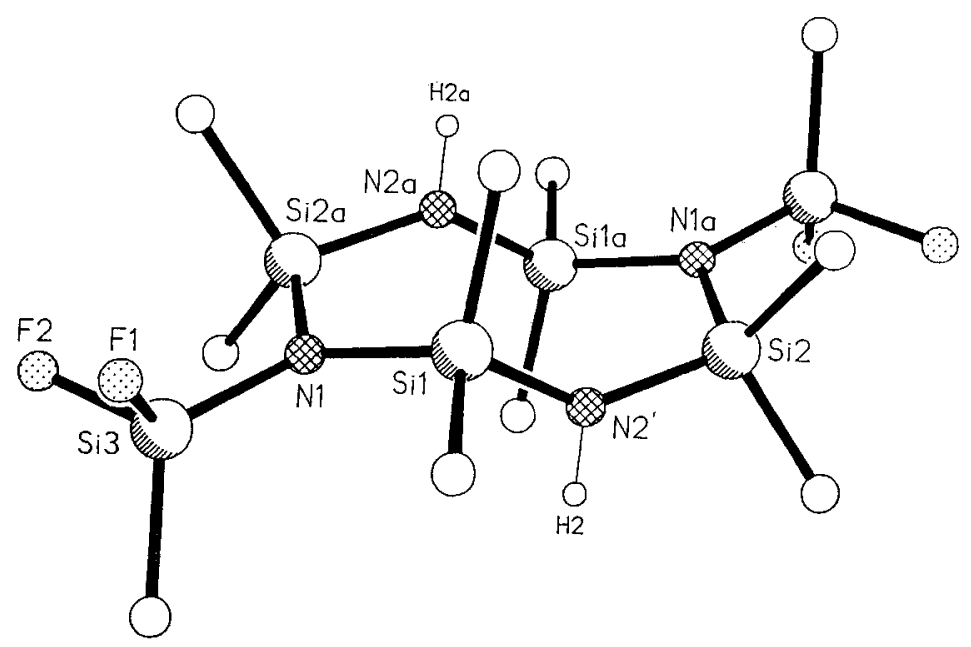

Abb. 5 Kristallstruktur von 8

(9) kurz, die endocyclischen Bindungen am N1-Atom sind mit einem Mittelwert von $177,0 \mathrm{pm}$ (8) bzw. 176,8 pm (9) entsprechend lang. Die Ringwinkel der $\mathrm{N}(\mathrm{H})$-Stickstoffatome sind mit $136,6^{\circ}(8) \mathrm{bzw} .135,7^{\circ}(9)$ deutlich aufgeweitet. Die $\mathrm{Si}-\mathrm{N}$-Si-Winkel der silylsubstituierten Stickstoffatome liegen auch hier bei einem Wert von $120^{\circ}$. Ein Vergleich der nichtbindenden Si-N-Abstände von 9 mit den entsprechenden Abständen im OMCTS [8] zeigt, daß die Disubstitution zu einer Verkürzung der nichtbindenden Abstände über den Ring führt. Während im OMCTS der kürzeste nichtbindende Abstand für die Sesselkonformation $360 \mathrm{pm}$ und für die Wannenkonformation $353 \mathrm{pm}$ beträgt, ist der Abstand von N2-Atom zum Sila-Atom in 9 nur 316,9 pm.

Obwohl beide Verbindungen im Kristall die gleiche Molekülstruktur annehmen, unterscheiden sie sich in der relativen Anordnung zueinander. Die Bis(difluormethyl)-substituierte Verbindung 8 bildet, wie auch die entsprechende monosubstituierte Verbindung 2, intermolekulare Wasserstoffbrücken im Kristall aus 
(Abb. 6). Dabei tritt ein Fluoratom jeder Silylgruppe mit einem Wasserstoffatom eines weiteren Moleküls in Wechselwirkung. Die benachbarten Moleküle sind dabei jeweils um $90^{\circ}$ gegeneinander verdreht, so daß ein parkettartiges

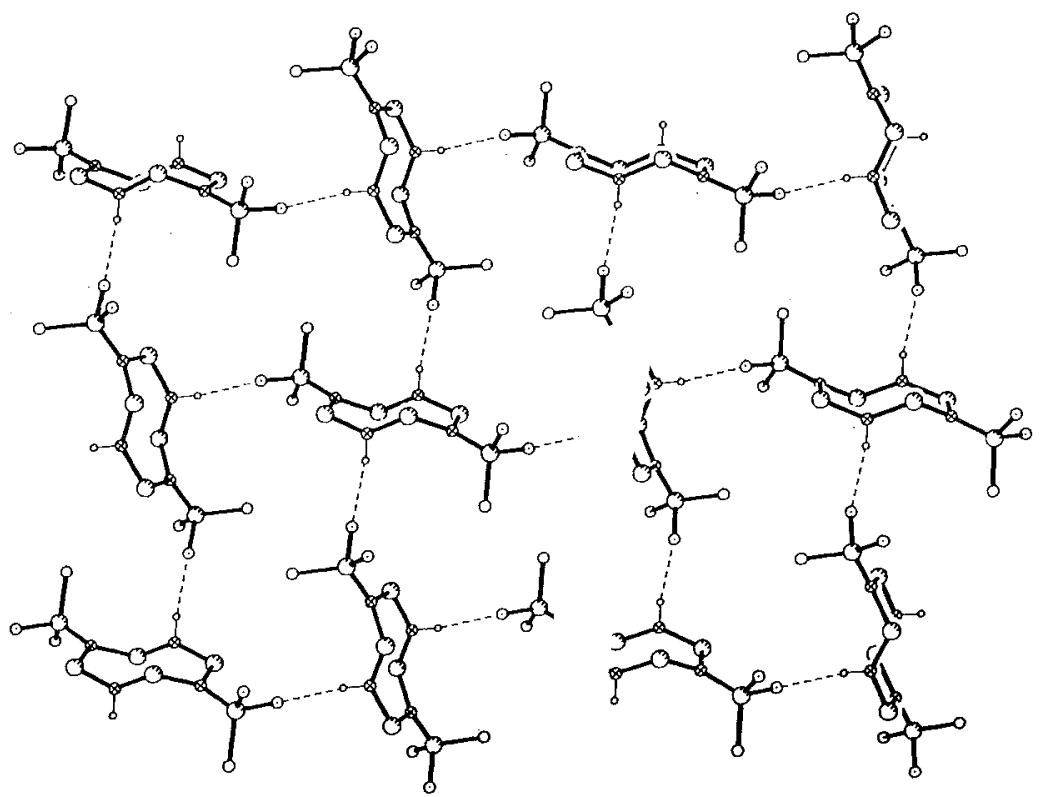

Abb. 6 Anordnung von 8 im Kristall

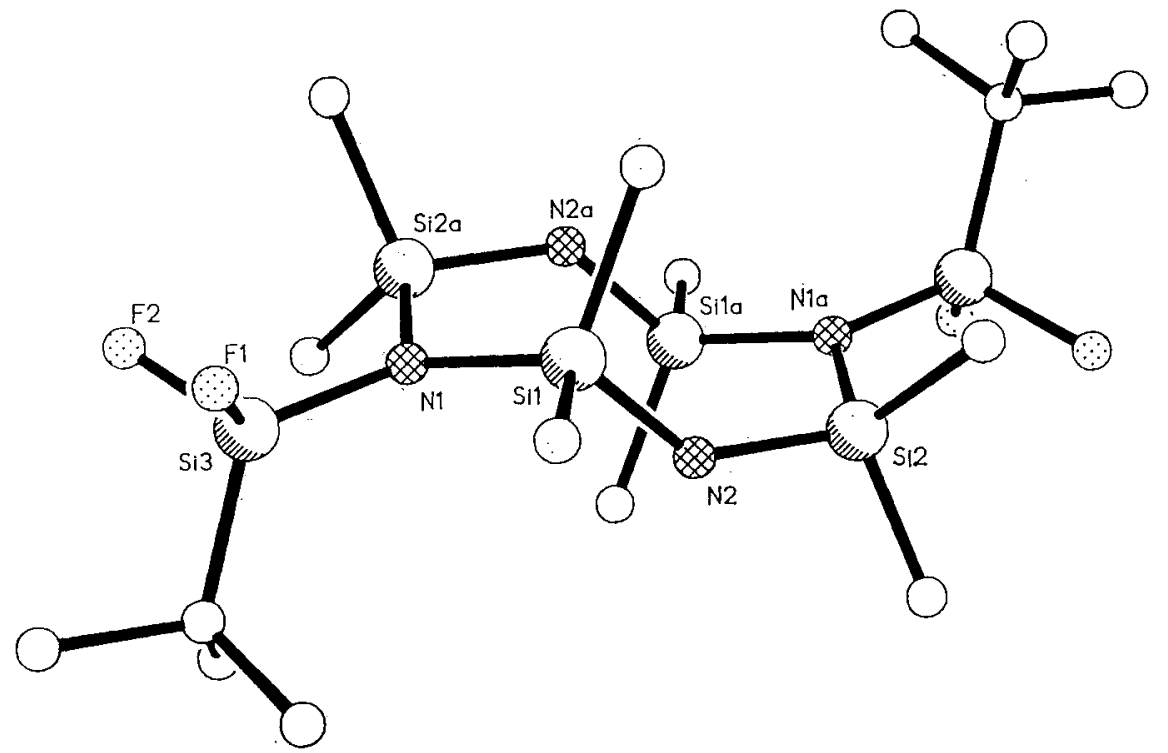

Abb. 7 Kristallstruktur von 9 
Schichtmuster aus zueinander versetzten Rechtecken entsteht. Der F-H-Abstand ist mit $232 \mathrm{pm}$ wiederum recht kurz.

Die Moleküle der Bis(tert.-butyldifluorsilyl)-substituierten Verbindung 9 zeigen dagegen in der Packungsdarstellung keine Anordnung, die auf intermolekulare H-F-Brücken schließen läßt. Die Moleküle sind durch Translation ineinander überführbar. Auch die betreffenden $\mathrm{H}-\mathrm{F}$-Abstände sprechen gegen derartige Wechselwirkungen. Das Fehlen von intermolekularen $\mathrm{H}-\mathrm{F}$-Wechselwirkungen bei 9 kann durch den größeren sterischen Anspruch einer voluminösen tert.-Butylgruppe im Vergleich zu einer Methylgruppe erklärt werden.

Kristallstruktur des Bicyclus 14 [6]

Das bicyclische System besteht aus zwei $\mathrm{Si}-\mathrm{N}$-Sechsringen. Die verbrückten Stickstoffatome N2 und N4 bilden mit den Atomen Si1, N1 und Si4 eine planare Anordnung. Die Atome N2, Si3, Si2 und N4 bilden ebenfalls eine Ebene. Im Gegensatz zum N1-Atom ragt das N3-Atom aus dieser Ebene heraus und ist dem Phenylring zugewandt. Der durch die Atome Si5, N2, Si3, N3, Si2, N4 definierte Sechsring nimmt somit eine Wannenkonformation ein. Die Konformation des durch die Atome Si5, N2, Si1, N1, Si4, N4 erhaltenen Sechsrings kann als „Halbsessel" beschrieben werden. Die zwei durch die Atomquartette N2, Si1, Si4, N4 und N2, Si3, Si2, N4 gebildeten Ebenen sind an der Linie N2-N4 im Winkel

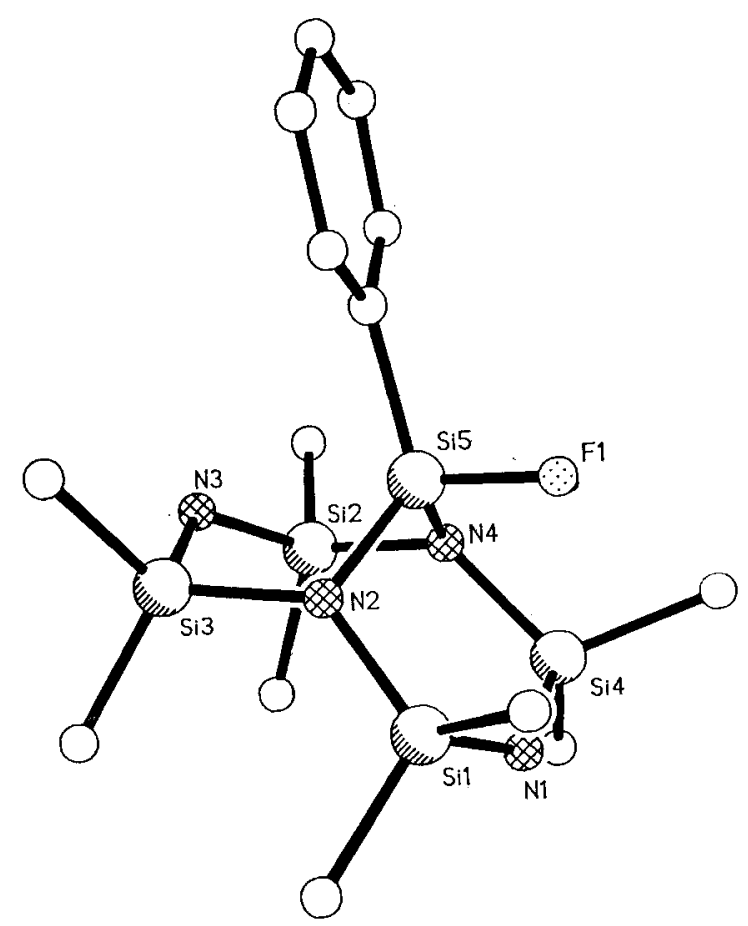

Abb. 8 Kristallstruktur von $\mathbf{1 4}$ 
von $130,1^{\circ}$ gegeneinander geneigt. Die Summe der Bindungswinkel an den dreifach silylsubstituierten Stickstoffatomen $\mathrm{N} 2$ und $\mathrm{N} 4$ betragen $350,2^{\circ}$ bzw. $342,2^{\circ}$; dies entspricht einer Abweichung aus der Ebene der umgebenden Siliciumatome von $31,3 \mathrm{pm}$ für N2 und $60,9 \mathrm{pm}$ für N4. Die Bindungsgeometrie der Stickstoffatome N2 und $\mathrm{N} 4$ ist folglich pyramidal. Wie die durchschnittliche Winkelsumme für bisher untersuchte trisilylsubstituierte Stickstoffatome (CCDC data base, 27 entries, 62 Winkelsummen, Mittelwert: $358,6^{\circ}$ ) zeigt, ist diese Geometrie für Silazane sehr ungewöhnlich. Der bisher kleinste Wert beträgt $350,6^{\circ}$ und wurde in der Struktur eines 1,3,5, 7-Tetraaza-2, 4, 6, 8-tetra-silabicyclo[4.2.0]octans gefunden [9].

Die Frage, warum das N3-Atom dem Phenylring zugeneigt, ist kann anhand von sterischen Überlegungen geklärt werden. Sowohl die Ausbildung einer planaren Anordnung mit den Atomen $\mathrm{Si} 3, \mathrm{Si} 2, \mathrm{~N} 2$ und $\mathrm{N} 4$ als auch das vollständige Umklappen des N3-Atoms zur Sesselkonformation des betreffenden Sechsrings führt zu einer zunehmenden sterischen Hinderung der an das Si2- und Si3-Atom gebundenen Methylgruppen mit dem Phenylring.

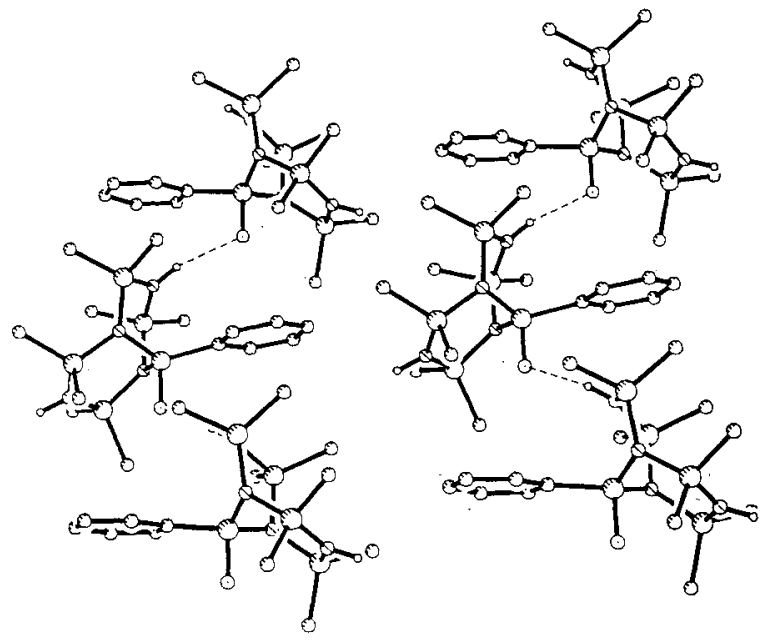

Abb. 9 Anordnung von $14 \mathrm{im}$ Kristall

14 bildet im kristallinen Zustand ebenfalls intermolekulare $\mathrm{H}-\mathrm{F}$-Brücken aus (Abb. 9). Das Fluoratom bildet eine Brücke zu dem Wasserstoffatom des N3Atoms eines Nachbarmoleküls aus. Daraus resultiert eine kettenförmige Anordnung der Moleküle in der Packung. Der H-F-Abstand beträgt 249,6 pm.

Die ${ }^{13} \mathrm{C}$ - und ${ }^{1} \mathrm{H}-\mathrm{NMR}$-Aufnahmen von 14 und 15 zeigen, daß die überbrückende RSiF-Gruppe in Lösung nicht durchschwingt, sondern wie im kristallinen Zustand sterisch fixiert ist. Im ${ }^{13} \mathrm{C}-\mathrm{NMR}$-Spektrum werden dementsprechend für die Methylgruppen vier chemische Verschiebungen beobachtet, welche den axial und äquatorial stehenden Methylgruppen eines jeden Sechsrings paarweise zuzuordnen sind. Die ${ }^{1} \mathrm{H}$-NMR-Spektren zeigen für die Methylgruppen eine weitere 
Verdopplung der Resonanzen. Durch die Aufnahme eines ${ }^{19} \mathrm{~F}-\mathrm{BB}$-entkoppelten ${ }^{1} \mathrm{H}$-NMR-Spektrums [5] konnte belegt werden, daß die Aufspaltung der Resonanzen nicht durch $\mathrm{H}-\mathrm{F}$-Kopplungen hervorgerufen wird, sondern auf der Nichtäquivalenz der paarweise zugeordneten Methylgruppen beruht. Dieser Effekt wird der Pseudochiralität des überbrückenden Si5-Atoms zugeordnet. Für die beiden zu tiefem Feld verschobenen Signale wird eine Kopplung von jeweils $3,2 \mathrm{~Hz}$ (14) bzw. 4,1 Hz (15) beobachtet. Diese sind als Raumkopplungen aufzufassen und werden den Methylgruppen $\mathrm{C}(2) \mathrm{H}_{3}$ und $\mathrm{C}(8) \mathrm{H}_{3}$ zugeteilt, da sie, wie aus der Kristallstruktur zu ersehen ist, dem Fluoratom zugeneigt sind.

\section{Experimenteller Teil}

Die Versuche wurden unter Feuchtigkeitsausschluß durchgeführt. Massenspektren: $\mathrm{CH}-5$ Spektrometer, Varian. NMR-Spektren: $30 \%$ ige Lösungen in $\mathrm{CDCl}_{\mathbf{3}}(\mathbf{2}-\mathbf{5}, \mathbf{7}-\mathbf{1 1})$ bzw. $\mathrm{C}_{6} \mathrm{D}_{6}(\mathbf{1 3}-\mathbf{1 5})$, TMD, $\mathrm{C}_{6} \mathrm{~F}_{6}$ int. Bruker WP 80 oder AM 250 Kernresonanzgerät. Die Reinheit von $2-5$ und $7-15$ wurde Massen- und NMR-spektroskopisch überprüft. Von 2, 3, 8, 9 and 15 liegen zusätzlich Röntgenstrukturanalysen vor.

Monosubstituierte Cyelotetrasilazane 2-5. $0,1 \mathrm{ml}$ OMCTS in $300 \mathrm{~cm}^{3} \mathrm{n}$-Hexan werden mit 0,1 mol $\mathrm{n}$-Butyllithium ( $15 \%$ ig in $\mathrm{n}$-Hexan) lithiiert und danach etwa $1 \mathrm{~h}$ unter Rühren zum Sieden erhitzt. Bei der Darstellung von 3-5 werden nach beendeter Butanabspaltung $20 \mathrm{~cm}^{3} \mathrm{THF}$ hinzugegeben. Anschließend wird die Aufschlämmung auf $-50^{\circ} \mathrm{C}$ abgekühlt (Innenthermometer) und $0,1 \mathrm{~mol}$ des entsprechenden Fluorsilans langsam eingeleitet bzw. hinzugetropft. Die Lösung wird innerhalb von $12 \mathrm{~h}$ auf Raumtemperatur gebracht. Nach Einengen der Lösung wird das Reaktionsgemisch durch Einkondensieren der flüchtigen Bestandteile in eine Kühlfalle von Lithiumfluorid abgetrennt. Das rückgebildete OMCTS wird durch Sublimation entfernt. 2-5 werden durch Destillation über eine $50 \mathrm{~cm}$-Vigreux-Kolonne von der disubstituierten Verbindung abgetrennt und nach mehrmaligem Umkristallisieren aus $\mathrm{n}$-Hexan rein erhalten.

1-Difluormethylsilyl-2,2,4,4,6,6,8,8-octamethylcyclotetrasilazan, 2. Fp. $62^{\circ} \mathrm{C}$. Ausbeute $3,7 \mathrm{~g}(10 \%)$.

MS: $\mathrm{m} / \mathrm{z}=357(100 \%)\left[\mathrm{M}-\mathrm{CH}_{3}\right]^{+}$, FI-Messung: $\mathrm{m} / \mathrm{z}=372(100 \%) \mathrm{M}^{+}$.

${ }^{1} \mathrm{H}-\mathrm{NMR}: 0,09 \mathrm{SiMe}_{2}, 0,26 \mathrm{SiMe}_{2}\left({ }^{5} \mathrm{~J}_{\mathrm{HF}}=1,1 \mathrm{~Hz}\right) .0,36 \mathrm{SiMeF}_{2}\left({ }^{3} \mathrm{~J}_{\mathrm{HF}}=5,1 \mathrm{~Hz}\right)$.

${ }^{13} \mathrm{C}-\mathrm{NMR}:-2,19 \mathrm{SiCF}_{2}\left({ }^{2} \mathrm{~J}_{\mathrm{CF}}=25,7 \mathrm{~Hz}\right), 3,37 \mathrm{SiC}_{2}, 4,86 \mathrm{SiC}_{2}\left({ }^{4} J_{\mathrm{CF}}=2,1 \mathrm{~Hz}\right)$.

${ }^{19} \mathrm{~F}-\mathrm{NMR}: 42,34$.

${ }^{29} \mathrm{Si}-\mathrm{NMR}:-32,07 \mathrm{SiF}_{2}\left({ }^{1} \mathrm{~J}_{\mathrm{SiF}}=271,6 \mathrm{~Hz}\right),-7,68,-6,91\left({ }^{3} \mathrm{~J}_{\mathrm{SiF}}=2,0 \mathrm{~Hz}\right)$.

1-Fluordimethylsilyl-2,2,4,4,6,6,8,8-octamethyleyclotetrasilazan, 3. Fp. $64^{\circ} \mathrm{C}$. Ausbeute $2,6 \mathrm{~g}(7 \%)$.

MS (F. I.): $\mathrm{m} / \mathrm{z}=368(16 \%) \mathbf{M}^{+}$.

${ }^{1} \mathrm{H}-\mathrm{NMR}: 0,08 \mathrm{SiMe}_{2}, 0,23 \mathrm{SiMe}_{2}\left({ }^{5} \mathbf{J}_{\mathrm{HF}}=1,4 \mathrm{~Hz}\right), 0,27 \mathrm{SiMe}_{2} \mathrm{~F} \quad\left({ }^{3} \mathrm{~J}_{\mathrm{HF}}=7,6 \mathrm{~Hz}\right)$.

${ }^{13} \mathrm{C}-\mathrm{NMR}: 3,36 \mathrm{SiC}_{2} \mathbf{F}\left({ }^{2} J_{\mathrm{CF}}=18,6 \mathrm{~Hz}\right), 3,55\left(\mathrm{SiC}_{2}, 5,60 \mathrm{SiC}_{2}\left({ }^{4} \mathrm{~J}_{\mathrm{CF}}=2,9 \mathrm{~Hz}\right)\right.$.

${ }^{19}$ F-NMR: 29,26 .

${ }^{29} \mathrm{Si}-\mathrm{NMR}:-8,42\left({ }^{3} \mathrm{~J}_{\mathrm{SiF}}=5,5 \mathrm{~Hz}\right),-7,97,9,14\left({ }^{1} \mathrm{~J}_{\mathrm{SiF}}=266,4 \mathrm{~Hz}\right)$.

1-Difluorphenylsilyl-2,2,4,4,6,6,8,8-octamethyleyclotetrasilazan, 4. Fp. $115^{\circ} \mathrm{C}$. Ausbeute $2,2 \mathrm{~g}(6 \%)$.

MS $m / z=419(100 \%)\left[\mathrm{MI}-\mathrm{CH}_{3}\right]^{+}$.

${ }^{1} \mathrm{H}-\mathrm{NMR}: 0,08 \mathrm{SiMe}_{2}, 0,27 \mathrm{SiMe}_{\mathbf{2}}\left({ }^{5} \mathrm{~J}_{\mathrm{HF}}=1,1 \mathrm{~Hz}\right), 7,25-7,80 \mathrm{Ph}$.

${ }^{13} \mathrm{C}-\mathrm{NMR}: 3,36 \mathrm{SiC}_{2}, 4,81 \mathrm{SiC}_{2}\left({ }^{4} \mathrm{~J}_{\mathrm{HF}}=1,9 \mathrm{~Hz}\right), 128,21,130,60\left({ }^{2} \mathrm{~J}_{\mathrm{CF}}=28,3 \mathrm{~Hz}\right), 131,39,134,27$

$\left({ }^{3} \mathrm{~J}_{\mathrm{CH}}=1,3 \mathrm{~Hz}\right)$. 
${ }^{19}$ F-NMR: 35,21 .

${ }^{29} \mathrm{Si}-\mathrm{NMR}:-47,70 \mathrm{SiF}_{2}\left({ }^{1} \mathrm{~J}_{\mathrm{SiF}}=268,9 \mathrm{~Hz}\right),-8,44\left({ }^{3} \mathbf{J}_{\mathrm{SiF}}=1,5 \mathrm{~Hz}\right),-5,88$.

1-tert.-Butyldifluorsilyl-2,2,4,4,6,6,8,8-octamethylcyclotetrasilazan, 5. Fp. $75^{\circ} \mathrm{C}$. Ausbeute $3,3 \mathrm{~g}(8 \%)$.

MS: $\mathrm{m} / \mathrm{z}=399(100 \%)\left[\mathrm{M}-\mathrm{CH}_{3}\right]^{+}$, F.I.-Messung: $\mathrm{m} / \mathrm{z}=414(100 \%) \mathrm{M}^{+}$.

${ }^{1} \mathrm{H}-\mathrm{NMR}: 0,10 \mathrm{SiMe}_{2}, 0,31 \mathrm{SiMe}_{2}\left({ }^{5} \mathrm{~J}_{\mathrm{HF}}=1,3 \mathrm{~Hz}\right), 0,56 \mathrm{NH}, 1,04 \mathrm{CMe}_{3}\left({ }^{4} \mathrm{~J}_{\mathrm{HF}}=1,1 \mathrm{~Hz}\right)$.

${ }^{13} \mathrm{C}-\mathrm{NMR}: 3,25 \mathrm{SiC}_{2}, 5,42 \mathrm{SiC}_{2}\left({ }^{4} \mathrm{~J}_{\mathrm{CF}}=2,8 \mathrm{~Hz}\right), 18,44 \mathbf{C C}_{3}\left({ }^{2} \mathrm{~J}_{\mathrm{CF}}=22,3 \mathrm{~Hz}\right), 27,15 \mathrm{CC}_{3}$.

${ }^{19} \mathrm{~F}-\mathrm{NMR}: 31,90$.

${ }^{29} \mathrm{Si}-\mathrm{NMR}:-34,70 \mathrm{SiF}_{2}\left({ }^{1} \mathrm{~J}_{\mathrm{SiF}}=295,7 \mathrm{~Hz}\right),-8,35,-6,54$.

Disubstituierte Cyclotetrasilazane 7-11 und 13. Eine Lösung von $0,05 \mathrm{~mol} 6[6]$ in $250 \mathrm{~cm}^{3}$ $\mathrm{n}$-Hexan und $50 \mathrm{~cm}^{3} \mathrm{THF}$ wird auf $-40^{\circ} \mathrm{C}$ abgekühlt. Danach werden langsam $0,1 \mathrm{~mol}$ des jeweiligen Fluorsilans (7-12) bzw. Fluorborans (13) hinzugegeben. Das Reaktionsgemisch wird innerhalb von 2 h auf Raumtemperatur erwärmt. 13 kristallisiert aus der Reaktionslösung in Form quadra. tischer Blättchen aus. Die Kristalle werden durch Waschen mit $\mathrm{n}$-Hexan vom Lithiumfluorid gereinigt. Nach Einengen der Lösung und Entfernen des Lithiumfluorids kristallisieren 8, 9 und 11 ebenfalls aus der Lösung aus. 7 und 10 kristallisieren nach erfolgter Destillation.

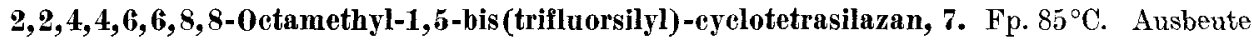
$18,0 \mathrm{~g}(78 \%)$.

MS: $\mathrm{m} / \mathrm{z}=445(100 \%)\left[\mathrm{M}-\mathrm{CH}_{3}\right]^{+}$.

${ }^{1} \mathrm{H}-\mathrm{NMR}: 0,31 \mathrm{SiMe}_{2}\left({ }^{5} \mathrm{~J}_{\mathrm{HF}}=1,0 \mathrm{~Hz}\right), 0,75 \mathrm{NH}$.

${ }^{13} \mathrm{C}: \mathrm{NMR}: 3,85 \mathrm{SiC}_{2}\left({ }^{4} \mathrm{~J}_{\mathrm{CF}}=1,5 \mathrm{~Hz}\right)$.

19F-NMR: 23,23 .

${ }^{29} \mathrm{Si}-\mathrm{NMR}$ : $-85,18 \mathrm{SiF}_{3}\left({ }^{1} \mathrm{~J}_{\mathrm{SiF}}=210,0 \mathrm{~Hz}\right),-3,93$.

1,5-Bis (difluormethylsilyl)-2,2,4,4,6,6,8,8-octamethyleyelotetrasilazan, 8. Fp. $70^{\circ} \mathrm{C}$. Ausbeute $20,8 \mathrm{~g}(92 \%)$.

MS: $\mathrm{m} / \mathrm{z}=437(100 \%)\left[\mathrm{M}-\mathrm{CH}_{3}\right]^{+}$, F.I.-Messung: $\mathrm{m} / \mathrm{z}=452(40 \%) \mathrm{M}^{+}$.

${ }^{1} \mathrm{H}-\mathrm{NMR}: 0,27 \mathrm{SiMe}_{2}\left({ }^{5} \mathrm{~J}_{\mathrm{HF}}=1,1 \mathrm{~Hz}\right), \quad 0,36 \mathrm{SiMeF}_{2}\left({ }^{3} \mathbf{J}_{\mathrm{HF}}=5,1 \mathrm{~Hz}\right)$.

${ }^{13} \mathrm{C}-\mathrm{NMR}:-2,37 \mathrm{SiCF}_{2}\left({ }^{2} \mathrm{~J}_{\mathrm{CF}}=25,5 \mathrm{~Hz}\right), 4,62 \mathrm{SiC}_{2}\left({ }^{4} \mathrm{~J}_{\mathrm{CF}}=1,9 \mathrm{~Hz}\right)$.

19F-NMR: 41,60 .

${ }^{29} \mathrm{Si}-\mathrm{NMR}:-32,07 \mathrm{SiF}_{\mathbf{2}}\left({ }^{1} \mathrm{~J}_{\mathrm{SiF}}=271,7 \mathrm{~Hz}\right),-6,38 \mathrm{SiMe}_{2}\left({ }^{3} \mathbf{J}_{\mathrm{SiF}}=\mathbf{2 , 1} \mathrm{Hz}\right)$.

1,5-Bis(tert.-butyldifluorsilyl)-2,2,4,4,6,6,8,8-octamethylcyclotetrasilazan, 9. Fp. $120^{\circ} \mathrm{C}$. Ausbeute $22,6 \mathrm{~g}(84 \%)$.

MS: $\mathrm{m} / \mathrm{z}=521(100 \%)\left[\mathrm{M}-\mathrm{CH}_{3}\right]^{+}$.

${ }^{1} \mathrm{H}-\mathrm{NMR}: 0,31 \mathrm{SiMe}_{2}\left({ }^{5} \mathrm{~J}_{\mathrm{HF}}=1,3 \mathrm{~Hz}\right), 0,63 \mathrm{NH}, 1,05 \mathrm{CMe}_{3}\left({ }^{4} \mathrm{~J}_{\mathrm{HF}}=1,1 \mathrm{~Hz}\right)$.

${ }^{13} \mathrm{C}-\mathrm{NMR}: 5,34 \mathrm{SiC}_{2}\left({ }^{4} \mathrm{~J}_{\mathrm{CF}}=2,6 \mathrm{~Hz}\right), 18,28 \mathrm{CC}_{3}\left({ }^{2} \mathrm{~J}_{\mathrm{CF}}=22,1 \mathrm{~Hz}\right), 27,13 \mathrm{CC}_{3}$.

${ }^{19} \mathrm{~F}-\mathrm{NMR}: 31,03$.

${ }^{29} \mathrm{Si}-\mathrm{NMR}:-34,93 \mathrm{SiF}_{2}\left({ }^{1} \mathrm{~J}_{\mathrm{SiF}}=296,4 \mathrm{~Hz}\right),-6,16\left({ }^{3} \mathrm{~J}_{\mathrm{SiF}}=2,1 \mathrm{~Hz}\right)$.

1,5-Bis(fluordimethyl)-2,2,4,4,6,6,8,8-octamethylcyclotetrasilazan, 10. Fp. $88^{\circ} \mathrm{C}$. Ausbeute $19,6 \mathrm{~g}(88 \%)$.

MS: $\mathrm{m} / \mathrm{z}=429(100 \%)\left[\mathrm{M}-\mathrm{CH}_{3}\right]^{+}$, F.I.-Messung: $\mathrm{m} / \mathrm{z}=444(30 \%) \mathrm{M}^{+}$.

${ }^{1} \mathrm{H}-\mathrm{NMR}: 0,23 \mathrm{SiMe}_{2}\left({ }^{5} \mathrm{~J}_{\mathrm{HF}}=1,3 \mathrm{~Hz}\right), 0,28 \mathrm{SiMe}_{2} \mathrm{~F}\left({ }^{3} \mathrm{~J}_{\mathrm{HF}}=7,5 \mathrm{~Hz}\right)$.

${ }^{13} \mathrm{C}-\mathrm{NMR}: 3,08 \mathrm{SiC}_{2} \mathrm{~F}\left({ }^{2} J_{\mathrm{CF}}=21,1 \mathrm{~Hz}\right), 5,53 \mathrm{SiC}_{2}\left({ }^{4} \mathrm{~J}_{\mathrm{CF}}=2,7 \mathrm{~Hz}\right)$.

${ }^{19} \mathrm{~F}-\mathrm{NMR}: 27,90$.

${ }^{29} \mathrm{Si}-\mathrm{NMR}:-8,22\left({ }^{3} \mathrm{~J}_{\mathrm{SiF}}=5,8 \mathrm{~Hz}\right), 9,41 \mathrm{SiF}\left({ }^{1} \mathrm{~J}_{\mathrm{SiF}}=266,0 \mathrm{~Hz}\right)$.

1,5-Bis (difluorisopropylsilyl) -2,2,4,4,6,6,8,8-0ctamethylcyclotetrasilazan, 11. Fp. $120^{\circ} \mathrm{C}$. Ausbeute $22,1 \mathrm{~g}(87 \%)$.

MS: $\mathrm{m} / \mathrm{z}=493(100 \%)\left[\mathrm{M}-\mathrm{CH}_{3}\right]^{+}$. 
${ }^{1} \mathrm{H}-\mathrm{NMR}: 0,27 \mathrm{SiMe}_{2}\left({ }^{5} \mathrm{~J}_{\mathrm{HF}}=1,1 \mathrm{~Hz}\right), 0,98-1,19 \mathrm{CHMe}_{2}$.

${ }^{13} \mathrm{C}-\mathrm{NMR}: 4,81 \mathrm{SiC}_{2}\left({ }^{4} \mathrm{~J}_{\mathrm{CF}}=1,9 \mathrm{~Hz}\right), 13,38 \mathrm{CC}_{2}\left({ }^{2} \mathrm{~J}_{\mathrm{CF}}=22,8 \mathrm{~Hz}\right), 16,70 \mathrm{CC}_{2}$.

${ }^{19}$ F-NMR: 29,79 .

${ }^{29} \mathrm{Si}-\mathrm{NMR}:-34,50 \mathrm{SiF}_{2}\left({ }^{1} \mathrm{~J}_{\mathrm{SiF}}=289,6 \mathrm{~Hz}\right),-6,64$.

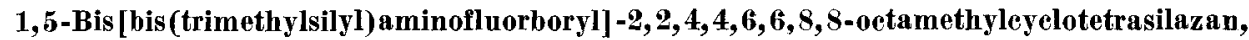
13. Fp. $205^{\circ} \mathrm{C}$. Ausbeute $26,8 \mathrm{~g}(80 \%)$.

MS: $\mathrm{m} / \mathrm{z}=655(38 \%)\left[\mathrm{M}-\mathrm{CH}_{3}\right]^{+}$, F.I.-Messung: $670(8 \%) \mathbf{M}^{+}$.

${ }^{1} \mathrm{H}-\mathrm{NMR}: 0,29 \mathrm{SiMe}_{3}\left({ }^{5} \mathrm{~J}_{\mathrm{HF}}=0,7 \mathrm{~Hz}\right), 0,41 \mathrm{SiMe}_{2}\left({ }^{5} \mathrm{~J}_{\mathrm{HF}}=1,1 \mathrm{~Hz}\right)$.

${ }^{11 B-N M R: ~} 27,50$.

${ }^{13} \mathrm{C}-\mathrm{NMR}: 3,46 \mathrm{SiC}_{3}\left({ }^{4} \mathrm{~J}_{\mathrm{CF}}=1,7 \mathrm{~Hz}\right), 4,35 \mathrm{SiC}_{2}\left({ }^{4} \mathrm{~J}_{\mathrm{CF}}=2,7 \mathrm{~Hz}\right)$.

${ }^{19} \mathrm{~F}-\mathrm{NMR}: 102,67$.

${ }^{29} \mathrm{Si}-\mathrm{NMR}:-7,61 \mathrm{SiMe}_{2}\left({ }^{3} \mathrm{~J}_{\mathrm{SiF}}=5,5 \mathrm{~Hz}\right), 3,94 \mathrm{SiMe}_{\mathbf{3}}\left({ }^{3} \mathrm{~J}_{\mathrm{SiF}^{\prime}}=\overline{5,5} \mathrm{~Hz}\right)$.

Bicyclen 14 und 15. $0,05 \mathrm{~mol} 6[6]$ in $250 \mathrm{~cm}^{3} \mathrm{n}$-Hexan und $50 \mathrm{~cm}^{3}$ THF werden bei $-40^{\circ} \mathrm{C}$ mit der äquimolaren Menge Phenyltrifluorsilan (14) bzw. tert.-Butyltrifluorsilan (15) versetzt. Nach Erwärmen auf Raumtemperatur wird das Rohprodukt vom Lithiumfluorid abgetrennt. Durch Umkristallisieren aus n-Hexan werden 14 und 15 rein erhalten.

9-Fluor-2,2, 4, 4,6,6,8,8-octamethyl-9-phenyl-1,3,5, 7-tetraaza-2,4,6,8,9-pentasila-

bicyelo[3.3.1] nonan, 14 [6]. Fp. $150^{\circ} \mathrm{C}$. Ausbeute $13,0 \mathrm{~g}(63 \%)$.

MS: $\mathrm{m} / \mathrm{z}=399(100 \%)\left[\mathrm{M}-\mathrm{CH}_{3}\right]^{+}$, F.I.-Messung: $414(100 \%) \mathrm{M}^{+}$.

${ }^{1} \mathrm{H}-\mathrm{NMR}: 0,020,0,022,0,223,0,225,0,284,0,286,0,470\left({ }^{5} \mathrm{~J}_{\mathrm{HF}}=3,2 \mathrm{~Hz}\right), 0,472\left({ }^{5} \mathrm{~J}_{\mathrm{HF}}=3,2 \mathrm{~Hz}\right)$ Me, 7,16-7,93 Ph.

${ }^{13} \mathrm{C}-\mathrm{NMR}: 4,09,6,34,7,83\left({ }^{4} \mathrm{~J}_{\mathrm{CF}}=1,0 \mathrm{~Hz}\right), 6,46\left({ }^{4} \mathrm{~J}_{\mathrm{CF}}=6,1 \mathrm{~Hz}\right)$ SiC, $127,64,129,86,134,31$ $\left({ }^{3} \mathrm{~J}_{\mathrm{CF}}=2,1 \mathrm{~Hz}\right) 137,94\left({ }^{2} \mathrm{~J}_{\mathrm{CF}}=25,0 \mathrm{~Hz}\right)$.

${ }^{19} \mathrm{~F} \cdot \mathrm{NMR}: 46,28$.

${ }^{29} \mathrm{Si}-\mathrm{NMR}:-31,91 \mathrm{SiF}\left({ }^{1} \mathrm{~J}_{\mathrm{SiF}}=263,7 \mathrm{~Hz}\right),-3,00\left({ }^{3} \mathrm{~J}_{\mathrm{SiF}}=4,7 \mathrm{~Hz}\right),-1,65\left({ }^{3} \mathrm{~J}_{\mathrm{SiF}}=4,0 \mathrm{~Hz}\right)$.

9-tert.-Butyl-9-fluor-2,2,4,4,6,6,8,8-octamethyl-1,3,5, 7-tetraaza-2, 4, 6,8,9-pentasilabicyclo[3.3.1]nonan, 15. Fp. $95^{\circ} \mathrm{C}$. Ausbeute $10,7 \mathrm{~g}(54 \%)$. $\mathrm{M}^{+}$.

MS: $\mathrm{m} / \mathrm{z}=379(48 \%)\left[\mathrm{M}-\mathrm{CH}_{3}\right]^{+}, 337(100 \%)\left[\mathrm{M}-\mathrm{C}_{4} \mathrm{H}_{9}\right]^{+}$, F.I.-Messung: $\mathrm{m} / \mathrm{z}=394(100 \%)$

${ }^{1} \mathrm{H}-\mathrm{NMR}: 0,088,0,089,0,150,0,27 \breve{\mathbf{5}}, 0,415\left({ }^{5} \mathrm{~J}_{\mathrm{HF}}=4,1 \mathrm{~Hz}\right), 0,425\left({ }^{5} \mathrm{~J}_{\mathrm{HF}}=4,1 \mathrm{~Hz}\right) \mathrm{Me}, 1,2 \mathrm{CMe}_{3}$ $\left({ }^{4} \mathrm{~J}_{\mathrm{HF}}=1,3 \mathrm{~Hz}\right)$.

${ }^{13} \mathrm{C}-\mathrm{NMR}: 4,79,6,34,8,01\left({ }^{4} \mathrm{~J}_{\mathrm{CF}}=0,9 \mathrm{~Hz}\right), 6,51\left({ }^{4} \mathrm{~J}_{\mathrm{CF}}=8,1 \mathrm{~Hz}\right) \mathrm{SiC}, 22,80 \quad \mathbf{C C}_{3}\left({ }^{2} \mathrm{~J}_{\mathrm{CF}}=18,3 \mathrm{~Hz}\right)$, $28,92 \mathrm{CC}_{3}\left({ }^{3} \mathrm{~J}_{\mathrm{CF}}=1,9 \mathrm{~Hz}\right)$.

${ }^{19} \mathrm{~F}-\mathrm{NMR}: 36,01$.

${ }^{29} \mathrm{Si}-\mathrm{N} M R:-18,93 \mathrm{SiF}\left({ }^{1} \mathrm{~J}_{\mathrm{SiF}}=269,2 \mathrm{~Hz}\right),-3,64\left({ }^{3} \mathrm{~J}_{\mathrm{SiF}}=6,9 \mathrm{~Hz}\right),-2,20\left({ }^{3} \mathrm{~J}_{\mathrm{SiF}}=6,4 \mathrm{~Hz}\right)$.

Kristallstrukturanalysen von 2, 3, 8, 9 und 14 [10]

Die Intensitätsmessungen aller vorgestellten Strukturen wurden auf einem Siemens-AED-Vierkreisdiffraktometer bei graphitmonochromatisierter MoK $\alpha$-Strahlung $(\lambda=71,069 \mathrm{pm})$ durchgeführt. Für $\mathbf{2}, 3$ und 8 erfolgte die Messung bei $-85^{\circ}$, für 9 und 14 bei Raumtemperatur. Die Strukturen wurden durch Direkte Methoden gelöst und nach dem Kleinste-Quadrate-Verfahren verfeinert. Wasserstoffatome wurden geometrisch ideal positioniert und mit festen Thermalparametern nach dem Reitermodell verfeinert. Bei 3, 8 und 9 wurden die $\mathrm{N}-\mathrm{H}$-Wasserstoffatome in der Differenz-FourierSynthese aufgefunden und isotrop verfeinert.

Kristallographische Daten. 2. Kristallgröße $0,5 \times 0,3 \times 0,2 \mathrm{~mm}^{3}$, Raumgruppe $\mathrm{P2} / \mathrm{n}$, $a=880,7(1), \quad b=1769,7(1), \quad c=1315,0(1) \mathrm{pm}, \quad \beta=93,47(1)^{\circ}, \quad V=2,046 \mathrm{~nm}^{3}, \quad Z=4, D_{\text {ber }}=$ $1,210 \mathrm{Mg} \mathrm{m}^{-3}, \mu=0,35 \mathrm{~mm}^{-1}$, Anzahl der gemessenen Reflexe $\left(2 \Theta_{\max }=50^{\circ}\right) 4085$, unabhängige 3581 , beobrehtete $[F>3 \sigma(F)] 2928, R=0,048, R_{w}=0,044\left[w^{-1}=\sigma^{2}(F)+0,0004 \cdot F^{2}\right]$, ver- 
Tabelle 1 Atomkoordinaten $\left(\times 10^{4}\right)$ und äquivalente isotrope Thermalparameter $\left(\mathrm{pm}^{2} \times 10^{-1}\right)$ von 2

\begin{tabular}{|c|c|c|c|c|}
\hline Atom & $x$ & $\mathrm{y}$ & $\mathbf{z}$ & $\left.\mathrm{U}(\mathrm{eq})^{\mathrm{a}}\right)$ \\
\hline Si(1) & $1182(1)$ & $8176(1)$ & $6200(1)$ & $24(1)$ \\
\hline $\mathrm{Si}(2)$ & $-1714(1)$ & $7427(1)$ & $6912(1)$ & $23(1)$ \\
\hline $\mathrm{Si}(3)$ & $-2972(1)$ & $9101(1)$ & $6564(1)$ & $23(1)$ \\
\hline $\mathrm{Si}(4)$ & $147(1)$ & $9442(1)$ & $7673(1)$ & $29(1)$ \\
\hline $\mathrm{Si}(5)$ & $-542(1)$ & $7032(1)$ & $4915(1)$ & $34(1)$ \\
\hline$N(1)$ & $-441(3)$ & $7592(1)$ & $5954(2)$ & $23(1)$ \\
\hline$N(2)$ & $-2717(3)$ & $8236(1)$ & $7145(2)$ & $25(1)$ \\
\hline$N(3)$ & $-1331(3)$ & $9607(1)$ & $6773(2)$ & $28(1)$ \\
\hline$N(4)$ & $1086(3)$ & $8627(1)$ & $7345(2)$ & $27(1)$ \\
\hline$F(1)$ & $628(3)$ & $7333(1)$ & $4134(1)$ & $55(1)$ \\
\hline$F(2)$ & $-2128(2)$ & $7111(1)$ & $4293(2)$ & $59(1)$ \\
\hline$C(11)$ & $2896(3)$ & $7558(2)$ & $6274(3)$ & 41(1) \\
\hline$C(12)$ & $1302(4)$ & $8886(2)$ & $5160(2)$ & $39(1)$ \\
\hline$C(21)$ & $-720(4)$ & $7065(2)$ & $8105(2)$ & $41(1)$ \\
\hline $\mathrm{C}(22)$ & $-3121(4)$ & $6683(2)$ & $6510(3)$ & $47(1)$ \\
\hline$C(31)$ & $-4568(3)$ & $9581(2)$ & $7171(3)$ & $41(1)$ \\
\hline $\mathrm{C}(32)$ & $-3444(4)$ & $9024(2)$ & $5175(2)$ & $40(1)$ \\
\hline$C(\$ 1)$ & $-546(4)$ & $9295(2)$ & $8975(2)$ & $47(1)$ \\
\hline $\mathrm{C}(42)$ & $1430(4)$ & $10275(2)$ & $7703(3)$ & $52(1)$ \\
\hline$C(51)$ & $-128(6)$ & $6023(2)$ & $5094(3)$ & $66(2)$ \\
\hline
\end{tabular}

a) äquivalente isotrope $U$ berechnet als ein Drittel der Spur des orthogonalen $U_{i j}$ Tensors

Tabelle 2 Ausgewählte Bindungslängen [pm] und -winkel [ $\left.{ }^{\circ}\right]$ von 2

\begin{tabular}{llll}
\hline $\mathrm{Si}(1)-\mathrm{N}(1)$ & $177,8(2)$ & $\mathrm{N}(1)-\mathrm{Si}(1)-\mathrm{N}(4)$ & $110,4(1)$ \\
$\mathrm{Si}(1)-\mathrm{N}(4)$ & $171,0(3)$ & $\mathrm{N}(1)-\mathrm{Si}(2)-\mathrm{N}(2)$ & $110,2(1)$ \\
$\mathrm{Si}(2)-\mathrm{N}(1)$ & $176,1(2)$ & $\mathrm{N}(2)-\mathrm{Si}(3)-\mathrm{N}(3)$ & $107,8(1)$ \\
$\mathrm{Si}(2)-\mathrm{N}(2)$ & $172,0(2)$ & $\mathrm{N}(3)-\mathrm{Si}(4)-\mathrm{N}(4)$ & $108,6(1)$ \\
$\mathrm{Si}(3)-\mathrm{N}(2)$ & $172,0(2)$ & $\mathrm{N}(1)-\mathrm{Si}(5)-\mathrm{F}(1)$ & $108,8(1)$ \\
$\mathrm{Si}(3)-\mathrm{N}(3)$ & $172,3(2)$ & $\mathrm{N}(1)-\mathrm{Si}(5)-\mathrm{F}(2)$ & $111,6(1)$ \\
$\mathrm{Si}(4)-\mathrm{N}(3)$ & $171,8(2)$ & $\mathrm{F}(1)-\mathrm{Si}(5)-\mathrm{F}(2)$ & $102,9(1)$ \\
$\mathrm{Si}(4)-\mathrm{N}(4)$ & $173,1(2)$ & $\mathrm{Si}(1)-\mathrm{N}(1)-\mathrm{Si}(2)$ & $120,4(1)$ \\
$\mathrm{Si}(5)-\mathrm{N}(1)$ & $168,5(2)$ & $\mathrm{Si}(1)-\mathrm{N}(1)-\mathrm{Si}(5)$ & $11.9,4(1)$ \\
$\mathrm{Si}(5)-\mathrm{F}(1)$ & $159,0(2)$ & $\mathrm{Si}(2)-\mathrm{N}(1)-\mathrm{Si}(5)$ & $118,6(1)$ \\
$\mathrm{Si}(5)-\mathrm{F}(2)$ & $158,2(2)$ & $\mathrm{Si}(2)-\mathrm{N}(2)-\mathrm{Si}(3)$ & $135,8(1)$ \\
& & $\mathrm{Si}(3)-\mathrm{N}(3)-\mathrm{Si}(4)$ & $127,9(1)$ \\
& & $\mathrm{Si}(1)-\mathrm{N}(4)-\mathrm{Si}(4)$ & $131,2(1)$ \\
\hline
\end{tabular}

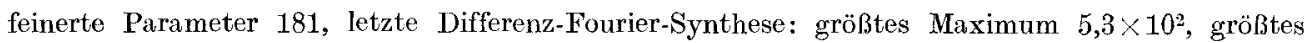
Minimum $3,1 \times 10^{2} \mathrm{e}^{-} \mathrm{nm}^{-3}$.

3: Kristallgrößc $0,6 \times 0,6 \times 0,1 \mathrm{~mm}^{3}$, Raumgruppe $\mathrm{P} 2_{1} / \mathrm{n}, \mathrm{a}=889,1(1), \mathrm{b}=1744,5(1), \mathrm{c}=$ $1383,8(2) \mathrm{pm}, \beta=91,4^{\circ}, \mathrm{V}=2,145 \mathrm{~nm}^{3}, \mathrm{Z}=4, \mathrm{D}_{\mathrm{ber}}=1,142 \mathrm{Mg} \mathrm{m}^{-3}, \mu=0,33 \mathrm{~mm}^{-1}$, Anzahl der gemessenen Reflexe $\left(2 \Theta_{\max }=50^{\circ}\right) 4752$, unabhängige 3639 , beobachtete $[\mathrm{F}>3 \sigma(\mathrm{F})] 3138, \mathrm{R}=$ $0,041, \mathbf{R}_{\mathrm{w}}=0,042\left[\mathrm{w}^{-1}=\sigma^{2}(\mathrm{~F})+0,0006 \cdot \mathrm{F}^{2}\right]$, verfeinerte Parameter 193, letzte Differenz-FourierSynthese: größtes Maximum 3,7 $\times 10^{2}$, größtes Minimum 2,7 $\times 10^{2} \mathrm{e}^{-} \mathrm{nm}^{-3}$.

8: Kristallgröße $0,7 \times 0,5 \times 0,4 \mathrm{~mm}^{3}$, Raumgruppe $\mathrm{P}_{1} / \mathrm{n}, \mathrm{a}=901,2(1), \quad \mathrm{b}=1174,1(1), \mathrm{e}=$ $1182,5(1) \mathrm{pm}, \beta=110,2(1)^{\circ}, \mathrm{V}=1,174 \mathrm{~nm}^{3}, \mathrm{Z}=2, \mathrm{D}_{\text {ber }}=1,281 \mathrm{Mg} \mathrm{m}^{-3}, \mu=0,38 \mathrm{~mm}^{-1}$, Anzahl 
Tabelle 3 Atomkoordinaten $\left(\times 10^{4}\right)$ und äquivalente isotrope Thermalparameter $\left(\mathrm{pm}^{2} \times 10^{-1}\right)$ von 3

\begin{tabular}{|c|c|c|c|c|}
\hline Atom & $\mathbf{x}$ & $\mathbf{y}$ & $\mathbf{z}$ & $\left.\mathbf{U}(e q)^{a}\right)$ \\
\hline $\mathrm{Si}(1)$ & $1434(1)$ & $6796(1)$ & $8815(1)$ & $25(1)$ \\
\hline $\mathrm{Si}(2)$ & $-1378(1)$ & $7590(1)$ & $8021(1)$ & $28(1)$ \\
\hline $\mathrm{Si}(3)$ & $-2801(1)$ & $5941(1)$ & $8353(1)$ & $28(1)$ \\
\hline $\mathrm{Si}(4)$ & $243(1)$ & $5439(1)$ & $7515(1)$ & $31(1)$ \\
\hline $\mathrm{Si}(5)$ & $26(1)$ & $8060(1)$ & $9925(1)$ & $35(1)$ \\
\hline$N(1)$ & $-100(2)$ & $7422(1)$ & $8977(1)$ & 24(1) \\
\hline$N(2)$ & $-2420(2)$ & $6781(1)$ & $7762(1)$ & $30(1)$ \\
\hline$N(3)$ & $-1239(2)$ & $5360(1)$ & $8277(1)$ & $31(1)$ \\
\hline$N(4)$ & $1199(2)$ & $6279(1)$ & $7768(1)$ & $29(1)$ \\
\hline $\mathbf{F}(\mathbf{1})$ & $1347(2)$ & $7750(1)$ & $10636(1)$ & $50(1)$ \\
\hline $\mathrm{C}(11)$ & 3175 (3) & $7381(2)$ & $8702(2)$ & $40(1)$ \\
\hline $\mathrm{C}(12)$ & $1595(3)$ & $6110(2)$ & $9837(2)$ & $41(1)$ \\
\hline $\mathrm{C}(21)$ & $-405(3)$ & $7927(2)$ & $6919(2)$ & $46(1)$ \\
\hline$C(22)$ & $-2727(3)$ & $8370(2)$ & $8324(2)$ & $56(1)$ \\
\hline $\mathrm{C}(\mathbf{3 1})$ & $-4455(3)$ & $5477(2)$ & $7741(2)$ & $45(1)$ \\
\hline $\mathrm{C}(32)$ & $-3218(3)$ & $6096(2)$ & $9649(2)$ & $45(1)$ \\
\hline$C(41)$ & $-352(4)$ & $548^{2}(2)$ & $6217(2)$ & $55(1)$ \\
\hline$C(42)$ & $1456(3)$ & $4579(2)$ & $7721(2)$ & $52(1)$ \\
\hline$C(51)$ & $689(4)$ & $9021(2)$ & $9580(2)$ & $61(1)$ \\
\hline$C(52)$ & $-1618(4)$ & $8099(3)$ & $10704(3)$ & $77(2)$ \\
\hline
\end{tabular}

a) äquivalente isotrope $U$ berechnet als ein Drittel der Spur des orthogonalen $V_{i j}$ Tensors

Tabelle 4 Ausgewählte Bindungslängen $[\mathrm{pm}]$ und -winkel $\left[{ }^{\circ}\right]$ von 3

\begin{tabular}{llll}
\hline $\mathrm{Si}(1)-\mathrm{N}(1)$ & $176,6(2)$ & $\mathrm{N}(1)-\mathrm{Si}(1)-\mathrm{N}(4)$ & $110,8(1)$ \\
$\mathrm{Si}(1)-\mathbf{N}(4)$ & $171,5(2)$ & $\mathrm{N}(1)-\mathrm{Si}(2)-\mathrm{N}(2)$ & $110,9(1)$ \\
$\mathrm{Si}(2)-\mathrm{N}(1)$ & $174,7(2)$ & $\mathrm{N}(2)-\mathrm{Si}(3)-\mathrm{N}(3)$ & $107,7(1)$ \\
$\mathrm{Si}(2)-\mathbf{N}(2)$ & $172,1(2)$ & $\mathrm{N}(3)-\mathrm{Si}(4)-\mathrm{N}(4)$ & $109,0(1)$ \\
$\mathrm{Si}(3)-\mathbf{N}(2)$ & $171,6(2)$ & $\mathrm{N}(1)-\mathrm{Si}(5)-\mathbf{F}(1)$ & $106,4(1)$ \\
$\mathrm{Si}(3)-\mathrm{N}(3)$ & $172,4(2)$ & $\mathrm{Si}(1)-\mathrm{N}(1)-\mathrm{Si}(2)$ & $119,8(1)$ \\
$\mathrm{Si}(4)-\mathrm{N}(3)$ & $171,3(2)$ & $\mathrm{Si}(1)-\mathrm{N}(1)-\mathrm{Si}(5)$ & $117,4(1)$ \\
$\mathrm{Si}(4)-\mathrm{N}(4)$ & $172,6(2)$ & $\mathrm{Si}(2)-\mathrm{N}(1)-\mathrm{Si}(5)$ & $119,9(1)$ \\
$\mathrm{Si}(5)-\mathrm{N}(1)$ & $172,1(2)$ & $\mathrm{Si}(2)-\mathrm{N}(2)-\mathrm{Si}(3)$ & $135,5(1)$ \\
$\mathrm{Si}(5)-\mathrm{F}(1)$ & $160,7(2)$ & $\mathrm{Si}(3)-\mathrm{N}(3)-\mathrm{Si}(4)$ & $128,6(1)$ \\
$\mathrm{Si}(5)-\mathrm{C}(51)$ & $184,4(3)$ & $\mathrm{Si}(1)-\mathrm{N}(4)-\mathrm{Si}(4)$ & $131,8(1)$ \\
$\mathrm{Si}(5)-\mathrm{C}(52)$ & $\mathbf{1 8 3 , 8 ( 3 )}$ & & \\
\hline
\end{tabular}

Tabelle 5 Atomkoordinaten $\left(\times 10^{4}\right)$ und äquivalente isotrope Thermalparameter $\left(\mathrm{pm}^{2} \times 10^{-1}\right)$ von 8

\begin{tabular}{|c|c|c|c|c|}
\hline Atom & $\mathbf{x}$ & $\mathbf{y}$ & $\mathbf{z}$ & $\left.\mathbf{U}(e q)^{a}\right)$ \\
\hline $\mathrm{Si}(\mathbf{1})$ & $1371(1)$ & $5416(1)$ & $3996(1)$ & $22(1)$ \\
\hline $\mathrm{Si}(2)$ & $1796(1)$ & $3607(1)$ & $6105(1)$ & $22(1)$ \\
\hline $\mathrm{Si}(3)$ & $479(1)$ & $7819(1)$ & $3174(1)$ & $31(1)$ \\
\hline$N(1)$ & $86(1)$ & $6595(1)$ & $3761(1)$ & $24(1)$ \\
\hline $\mathbf{N}(2)$ & $1862(1)$ & $4902(1)$ & $5440(1)$ & $23(1)$ \\
\hline$F(1)$ & $887(2)$ & $7598(1)$ & $1990(1)$ & $49(1)$ \\
\hline$F(2)$ & $-1128(1)$ & $8530(1)$ & $2662(1)$ & $52(1)$ \\
\hline$C(11)$ & $3228(2)$ & $5883(2)$ & $3786(2)$ & $41(1)$ \\
\hline$C(12)$ & $439(2)$ & $4302(2)$ & $2868(2)$ & $34(1)$ \\
\hline$C(21)$ & $2208(2)$ & $2405(2)$ & $5231(2)$ & $33(1)$ \\
\hline$C(22)$ & $3329(2)$ & $3680(2)$ & $7640(2)$ & $32(1)$ \\
\hline C(31) & $1982(3)$ & $8767(2)$ & $4160(2)$ & $51(1)$ \\
\hline
\end{tabular}

a) aquivalente isotrope $U$ berechnet als ein Drittel der Spur des orthogonalen $U_{i j}$ Tensors 
Tabelle 6 Ausgewählte Bindungslängen $[\mathrm{pm}]$ und -winkel $\left[{ }^{\circ}\right]$ von 8

\begin{tabular}{llll}
\hline $\mathrm{Si}(1)-\mathrm{N}(1)$ & $176,4(1)$ & $\mathrm{N}(1)-\mathrm{Si}(1)-\mathrm{N}(2)$ & $111,2(1)$ \\
$\mathrm{Si}(1)-\mathrm{N}(2)$ & $171,9(1)$ & $\mathrm{N}(2)-\mathrm{Si}(2)-\mathrm{N}(1 \mathrm{a})$ & $110,1(1)$ \\
$\mathrm{Si}(2)-\mathrm{N}(2)$ & $172,2(1)$ & $\mathrm{N}(1)-\mathrm{Si}(3)-\mathrm{F}(1)$ & $111,7(1)$ \\
$\mathrm{Si}(2)-\mathrm{N}(1 \mathrm{a})$ & $177,3(1)$ & $\mathrm{N}(1)-\mathrm{Si}(3)-\mathrm{F}(2)$ & $108,0(1)$ \\
$\mathrm{Si}(3)-\mathrm{N}(1)$ & $168,5(1)$ & $\mathrm{F}(1)-\mathrm{Si}(3)-\mathrm{F}(2)$ & $101,9(1)$ \\
$\mathrm{Si}(3)-\mathrm{F}(1)$ & $158,7(2)$ & $\mathrm{Si}(1)-\mathrm{N}(1)-\mathrm{Si}(3)$ & $120,9(1)$ \\
$\mathrm{Si}(3)-\mathrm{F}(2)$ & $159,9(1)$ & $\mathrm{Si}(1)-\mathrm{N}(1)-\mathrm{Si}(2 a)$ & $118,5(1)$ \\
$\mathrm{N}(1)-\mathrm{Si}(2 \mathrm{a})$ & $177,3(1)$ & $\mathrm{Si}(3)-\mathrm{N}(1)-\mathrm{Si}(2 \mathrm{a})$ & $119,6(1)$ \\
& & $\mathrm{Si}(1)-\mathrm{N}(2)-\mathrm{Si}(2)$ & $136,6(1)$ \\
\hline
\end{tabular}

Tabelle 7 Atomkoordinaten $\left(\times 10^{4}\right)$ und äquivalente isotrope Thermalparameter $\left(\mathrm{pm}^{2} \times 10^{-1}\right)$ von 9

\begin{tabular}{|c|c|c|c|c|}
\hline Atom & $\mathrm{x}$ & $\mathrm{y}$ & z & $\left.\mathrm{U}(\mathrm{eq})^{\mathrm{a}}\right)$ \\
\hline $\mathbf{S i}(1)$ & $3902(1)$ & $9727(1)$ & $8593(1)$ & $40(1)$ \\
\hline $\mathbf{S i}(2)$ & $3449(1)$ & $9025(1)$ & $11903(2)$ & $44(1)$ \\
\hline $\mathrm{Si}(3)$ & $3895(1)$ & $12404(1)$ & $6536(1)$ & $43(1)$ \\
\hline $\mathrm{N}(1)$ & $4655(3)$ & $11152(3)$ & $7793(2)$ & $37(1)$ \\
\hline$N(2)$ & $3154(3)$ & $10100(3)$ & $10392(3)$ & $43(1)$ \\
\hline $\mathbf{F}(\mathbf{1})$ & $3457(3)$ & $11554(2)$ & $5464(2)$ & $67(1)$ \\
\hline $\mathbf{F}(2)$ & $5350(2)$ & $12830(2)$ & $5577(2)$ & $69(1)$ \\
\hline$C(1)$ & $2178(5)$ & $9796(4)$ & $7876(4)$ & $66(2)$ \\
\hline$C(2)$ & $5528(5)$ & $7695(4)$ & $8234(3)$ & $60(2)$ \\
\hline $\mathrm{C}(3)$ & $1716(4)$ & $10130(5)$ & $13395(3)$ & $70(2)$ \\
\hline $\mathrm{C}(4)$ & $3427(5)$ & $7065(4)$ & $11695(4)$ & $70(2)$ \\
\hline $\mathrm{C}(5)$ & $2097(4)$ & $14310(4)$ & $7049(4)$ & $58(2)$ \\
\hline $\mathrm{C}(6)$ & $2397(6)$ & $15302(5)$ & $8006(6)$ & $108(3)$ \\
\hline$C(7)$ & $522(5)$ & $14090(5)$ & $7796(5)$ & $97(2)$ \\
\hline$C(8)$ & $1839(6)$ & $15225(5)$ & $5682(5)$ & $103(3)$ \\
\hline
\end{tabular}

a) aquivalente isotrope U berechnet als ein Drittel der Spur des orthogonalen $U_{i j}$ Tensors

Tabelle 8 Ausgewählte Bindungslängen [pm] und -winkel $\left[{ }^{\circ}\right]$ von 9

\begin{tabular}{llll}
\hline $\mathrm{Si}(1)-\mathrm{N}(1)$ & $176,2(3)$ & $\mathrm{N}(1)-\mathrm{Si}(1)-\mathrm{N}(2)$ & $109,6(1)$ \\
$\mathrm{Si}(1)-\mathrm{N}(2)$ & $172,1(3)$ & $\mathrm{N}(1)-\mathrm{Si}(3)-\mathrm{F}(1)$ & $111,3(1)$ \\
$\mathrm{Si}(2)-\mathrm{N}(2)$ & $172,3(3)$ & $\mathrm{N}(1)-\mathrm{Si}(3)-\mathrm{F}(2)$ & $107,4(1)$ \\
$\mathrm{Si}(2)-\mathrm{N}(1 \mathrm{a})$ & $177,4(3)$ & $\mathrm{F}(1)-\mathrm{Si}(3)-\mathrm{F}(2)$ & $103,6(1)$ \\
$\mathrm{Si}(3)-\mathrm{N}(1)$ & $169,5(2)$ & $\mathrm{Si}(1)-\mathrm{N}(1)-\mathrm{Si}(3)$ & $123,8(2)$ \\
$\mathrm{Si}(3)-\mathrm{F}(1)$ & $158,1(3)$ & $\mathrm{Si}(1)-\mathrm{N}(1)-\mathrm{Si}(2 a)$ & $116,1(1)$ \\
$\mathrm{Si}(3)-\mathrm{F}(2)$ & $158,6(2)$ & $\mathrm{Si}(3)-\mathrm{N}(1)-\mathrm{Si}(2 a)$ & $118,9(2)$ \\
$\mathrm{N}(1)-\mathrm{Si}(2 a)$ & $177,4(3)$ & $\mathrm{Si}(1)-\mathrm{N}(2)-\mathrm{Si}(2)$ & $135,7(1)$ \\
\hline
\end{tabular}

der gemessenen Reflexe $\left(2 \Theta_{\max }==45^{\circ}\right) 4350$, unabhängige 2061 , bcobachtete $[\mathrm{F}>3 \sigma(\mathrm{F})] 2014$, $\mathbf{R}=0,030, \mathbf{R}_{\mathbf{w}}=0,041\left[\mathbf{w}^{-1}=\sigma^{2}(\mathrm{~F})+0,0008 \cdot \mathbf{F}^{2}\right]$, verfeinerte Parameter 11.2, letzte DifferenzFourier-Synthese: größtes Maximum 2,6 $6 \times 10^{2}$, größtes Minimum 2,5 $\times 10^{2} \mathrm{e}^{-\mathrm{nm}^{-3}}$.

9: Kristallgröße $0,2 \times 0,3 \times 0,2 \mathrm{~mm}^{3}$, Raumgruppe $\mathrm{P}-1, \mathrm{a}=914,3(5), \mathrm{b}=927,9(5), \mathrm{c}=$ $976,0(5) \mathrm{pm}, \quad x=84,88(2)^{\circ}, \beta=74,94(2)^{\circ}, \gamma=66,06(2)^{\circ}, \mathrm{V}=0,731 \mathrm{~nm}^{3}, \mathrm{Z}=1, \mathrm{D}_{\mathrm{ber}}=$ $1,221 \mathrm{Mg} \mathrm{m}^{-3}, \mu=0,31 \mathrm{~mm}^{-1}$, Anzahl der gemessenen Reflexe $\left(2 \Theta_{\max }=45^{\circ}\right) 1922$, unabhängige 1903 , beobachtete $[\mathbf{F}>3 \sigma(\mathrm{F})] 1601, \mathbf{R}=0,038, \mathbf{R}_{\mathbf{w}}=0,039\left[\mathbf{w}^{-1}=\sigma^{2}(\mathbf{F})+0,0003 \cdot \mathbf{F}^{2}\right]$, verfeinerte Parameter 139, letzte Differenz-Fourier-Synthese: größtes Maximum 2,3 $\times 10^{2}$, größtes Minimum $2,0 \times 10^{2} \mathrm{e}^{-} \mathrm{nm}^{-3}$. 
Tabelle 9 Atomkoordinaten $\left(\times 10^{4}\right)$ und äquivalente isotrope Thermalparameter $\left(\mathrm{pm}^{2} \times 10^{-1}\right)$ von 14

\begin{tabular}{|c|c|c|c|c|}
\hline Atom & $\mathbf{x}$ & $\mathrm{y}$ & $\mathbf{z}$ & $\mathrm{U}(\mathrm{eq})^{\mathrm{a})}$ \\
\hline $\mathrm{Si}(1)$ & 8200 & $8102(3)$ & 6800 & $42(2)$ \\
\hline $\mathrm{Si}(2)$ & $1978(3)$ & $6990(3)$ & $5315(3)$ & $51(2)$ \\
\hline $\operatorname{Si}(3)$ & $4002(4)$ & $6713(3)$ & $4439(3)$ & $51(2)$ \\
\hline $\mathrm{Si}(5)$ & $6219(3)$ & $6060(2)$ & $6760(3)$ & $38(2)$ \\
\hline $\mathrm{Si}(4)$ & $6204(4)$ & $8155(3)$ & $7764(3)$ & $48(3)$ \\
\hline$N(1)$ & $7620(11)$ & $8686(7)$ & $7518(9)$ & $53(7)$ \\
\hline$N(2)$ & $6468(8)$ & $6874(7)$ & $5976(7)$ & $40(6)$ \\
\hline$N(3)$ & $2229(9)$ & $6313(7)$ & $4484(7)$ & $44(7)$ \\
\hline$N(4)$ & $4664(9)$ & $6934(7)$ & $6768(7)$ & $38(6)$ \\
\hline $\mathbf{F}(1)$ & $8646(7)$ & $6057(5)$ & $8123(5)$ & $54(5)$ \\
\hline $\mathrm{C}(1)$ & $7873(16)$ & $9300(9)$ & $5882(13)$ & $71(12)$ \\
\hline$C(2)$ & $11135(13)$ & $7650(11)$ & $7928(11)$ & $67(10)$ \\
\hline$C(3)$ & $894(17)$ & $8560(12)$ & $4736(13)$ & $101(14)$ \\
\hline$C(4)$ & $11(13)$ & $6154(13)$ & $5142(12)$ & $76(10)$ \\
\hline$C(5)$ & $4058(13)$ & $5463(12)$ & $3710(11)$ & $79(10)$ \\
\hline$C(6)$ & $3085(18)$ & $8094(14)$ & $3530(12)$ & $110(13)$ \\
\hline$C(7)$ & $4534(13)$ & $9388(11)$ & $7534(12)$ & $65(12)$ \\
\hline $\mathrm{C}(8)$ & $8026(14)$ & $7658(11)$ & $9339(11)$ & $70(12)$ \\
\hline $\mathrm{C}(9)$ & $5707(12)$ & $4410(9)$ & $6480(9)$ & $37(8)$ \\
\hline$C(10)$ & $4474(14)$ & $3797(11)$ & $6526(12)$ & $57(10)$ \\
\hline $\mathrm{C}(11)$ & $4325(16)$ & $2551(11)$ & $6422(13)$ & $71(12)$ \\
\hline$C(12)$ & $5401(15)$ & $1916(12)$ & $6275(12)$ & $68(11)$ \\
\hline $\mathrm{C}(13)$ & $6609(16)$ & $2506(12)$ & $6220(13)$ & $54(10)$ \\
\hline $\mathrm{C}(14)$ & $6809(15)$ & $3760(11)$ & $6358(11)$ & $54(10)$ \\
\hline
\end{tabular}

a) äquivalente isotrope $U$ berechnet als ein Drittel der Spur des orthogonalen $U_{i j}$ Tensors

Tabelle 10 Ausgewählte Bindungslängen $[\mathrm{pm}]$ und -winkel $\left[{ }^{\circ}\right]$ von 14

\begin{tabular}{llll}
\hline $\mathrm{Si}(1)-\mathrm{N}(1)$ & $174,7(15)$ & $\mathrm{N}(1)-\mathrm{Si}(1)-\mathrm{N}(2)$ & $105,8(5)$ \\
$\mathrm{Si}(1)-\mathrm{N}(2)$ & $173,5(7)$ & $\mathrm{N}(3)-\mathrm{Si}(2)-\mathrm{N}(4)$ & $102,1(5)$ \\
$\mathrm{Si}(2)-\mathrm{N}(3)$ & $172,8(14)$ & $\mathrm{N}(2)-\mathrm{Si}(3)-\mathrm{N}(3)$ & $100,9(6)$ \\
$\mathrm{Si}(2)-\mathrm{N}(4)$ & $174,3(5)$ & $\mathrm{N}(2)-\mathrm{Si}(5)-\mathrm{N}(4)$ & $106,1(5)$ \\
$\mathrm{Si}(3)-\mathrm{N}(2)$ & $175,3(7)$ & $\mathrm{N}(2)-\mathrm{Si}(5)-\mathrm{F}(1)$ & $104,5(4)$ \\
$\mathrm{Si}(3)-\mathrm{N}(3)$ & $172,8(13)$ & $\mathrm{N}(4)-\mathrm{Si}(5)-\mathrm{F}(1)$ & $105,5(5)$ \\
$\mathrm{Si}(4)-\mathrm{N}(1)$ & $171,8(16)$ & $\mathrm{N}(1)-\mathrm{Si}(4)-\mathrm{N}(4)$ & $104,8(6)$ \\
$\mathrm{Si}(4)-\mathrm{N}(4)$ & $175,8(8)$ & $\mathrm{Si}(1)-\mathrm{N}(1)-\mathrm{Si}(4)$ & $133,9(5)$ \\
$\mathrm{Si}(5)-\mathrm{N}(2)$ & $173,2(13)$ & $\mathrm{Si}(1)-\mathrm{N}(2)-\mathrm{Si}(3)$ & $131,2(5)$ \\
$\mathrm{Si}(5)-\mathrm{N}(4)$ & $172,4(12)$ & $\mathrm{Si}(1)-\mathrm{N}(2)-\mathrm{Si}(5)$ & $111,5(6)$ \\
$\mathrm{Si}(5)-\mathrm{F}(1)$ & $160,4(5)$ & $\mathrm{Si}(3)-\mathrm{N}(2)-\mathrm{Si}(5)$ & $107,5(4)$ \\
& & $\mathrm{Si}(2)-\mathrm{N}(3)-\mathrm{Si}(3)$ & $127,1(5)$ \\
& & $\mathrm{Si}(2)-\mathrm{N}(4)-\mathrm{Si}(5)$ & $108,9(6)$ \\
& & $\mathrm{Si}(2)-\mathrm{N}(4)-\mathrm{Si}(4)$ & $126,4(5)$ \\
& & $\mathrm{Si}(5)-\mathrm{N}(4)-\mathrm{Si}(4)$ & $110,8(4)$ \\
\hline
\end{tabular}

14 [6]: Kristallgröße $0,3 \times 0,2 \times 0,2 \mathrm{~mm}^{3}$, Raumgruppe $\mathrm{Pc}, \mathrm{a}=909,6(2), \mathrm{b}=1110,1(2), \mathrm{c}=$ $1659,8(3) \mathrm{pm}, \beta=137,97(2)^{\circ}, \mathrm{V}=1,122 \mathrm{~nm}^{3}, \mathrm{Z}=2, \mathrm{D}_{\text {ber }}=1,228 \mathrm{Mg} \mathrm{m}^{-3}, \mu=0,32 \mathrm{~mm}^{-1}$, Anzahl der gemessenen Reflexe $2\left(\Theta_{\max }=45^{\circ}\right)$ 1554, unabhängige 1463 , beobachtete $[F>3 \sigma(\mathrm{F})] 1033$, $\mathrm{R}==0,060, \mathbf{R}_{\mathrm{w}}=0,053\left[\mathrm{w}^{-1}=\sigma^{2}(\mathrm{~F})+0,0008 \cdot \mathbf{F}^{\mathrm{2}}\right]$, verfeinerte Parameter 215, letzte DifferenzFourier-Synthese: größtes Maximum $3,0 \times 10^{2}$, größtes Minimum $3,0 \times 10^{2} \mathrm{e}^{-} \mathrm{nm}^{-3}$.

Der Deutschen Forschungsgemeinschaft und dem Fonds der Chemischen Industrie danken wir für die Unterstützung dieser Arbeit. 


\section{Literatur}

[1] Brewer, S. D.; Haber, C. P.: J. Am. Chem. Soc. 79 (1948) 3888.

[2] Fink, W.: Angew. Chem. 78 (1966) 803.

[3] Kuingebiel, U.: Nachr. Chem. Tech. Lab. 35 (1987) 1042.

[4] Klingebiel, U.; Enterlivg, D.; Melleer, A.: Chem. Ber. 110 (1977) 1277.

[5] DIPPEL, K.: Dissertation, Univ. Göttingen 1989.

[6] Dippel, K.; Kitveebiel, U.; Noltemeyer, M.; Pader, F.; Sheldrick, G. M. : Angew. Chem. 100 (1988) 1093.

[7] Klingebiel, U.; Skoda, L.: Z. Naturforsch. 40b (1985) 913.

[8] Smith, G. S.; Alexander, L. E.: Acta Crystallogr. 16 (1963) 1015.

[9] Clegg, W.; Klingebiel, U.; Sheldrick, G. M.; Skoda, L.; Vater, N.: Z. Naturforsch. 35b (1980) 1503.

[10] Weitere Einzelheiten zu den Kristallstrukturanalysen können beim Fachinformationszentrum Energie, Physik, Mathematik, D-7514 Eggenstein-Leopoldshafen 2, unter Angabe der Hinterlegungsnummer CSD 54197, der Autoren und des Zeitschriftenzitats angefordert werden.

Bei der Redaktion eingegangen am 4. August 1989.

Anschr. d. Verf.: Dr. K. Dippel, Prof. Dr. U. Klingebiel, T. Kottke, F. Paugr, Prof. G. M. Sheldrick, Dr. D. Stalke, Inst. f. Anorg. Chemie d. Univ., Tammannstr. 4, D-3400 Göttingen 